\title{
Micro RNAs are minor constituents of extracellular vesicles and are hardly delivered to target cells
}

Manuel Albanese ${ }^{1,2,3 \dagger}$, Yen-Fu Adam Chen ${ }^{1,3 \dagger}$, Corinna Hüls ${ }^{1,3}$, Kathrin Gärtner ${ }^{1,3}$, Takanobu Tagawa $^{1,3,5}$, Ernesto Mejias-Perez ${ }^{2,3}$, Oliver T. Keppler ${ }^{2,3}$, Christine Göbel ${ }^{1,3}$, Reinhard Zeidler ${ }^{1,3,4}$, Mikhail Shein $^{6}$, Anne K. Schütz ${ }^{6,7}$ and Wolfgang Hammerschmidt ${ }^{1,3^{*}}$

${ }^{1}$ Research Unit Gene Vectors, Helmholtz Zentrum München, German Research Center for Environmental Health, Munich, Germany

${ }^{2}$ Max von Pettenkofer Institute and Gene Center, Virology, National Reference Center for Retroviruses, Faculty of Medicine, LMU München, Munich, Germany.

${ }^{3}$ German Centre for Infection Research (DZIF), Partner site Munich, Germany

${ }^{4}$ Department of Otorhinolaryngology, Klinikum der Universität München, Marchioninistr. 15, 81377 Munich, Germany

${ }^{5}$ Current address: HIV and AIDS Malignancy Branch, Center for Cancer Research, National Cancer Institute, National Institutes of Health, Bethesda, Maryland, USA

${ }^{6}$ Bavarian NMR Center, Department of Chemistry, Technical University of Munich, 85748, Garching, Germany.

${ }^{7}$ Institute of Structural Biology, Helmholtz Zentrum München, 85764 Neuherberg, Germany.

${ }^{\dagger}$ shared authorship

*Corresponding author

Wolfgang Hammerschmidt

Marchioninistrasse 25

D-81377 Muenchen

Germany

Phone: $+49-89-3187-1506$

Fax: +49-89-3187-4225

E-mail: hammerschmidt@helmholtz-muenchen.de

Keywords: extracellular vesicles, herpesvirus, microRNA, EV fusion assay 


\begin{abstract}
Mammalian cells release different types of vesicles, collectively termed extracellular vesicles (EVs). EVs contain cellular microRNAs (miRNAs) with an apparent potential to deliver their miRNA cargo to recipient cells to affect the stability of individual mRNAs and the cells' transcriptome. The extent to which miRNAs are exported via the EV route and whether they contribute to cell-cell communication are controversial. To address these issues, we analyzed the capacity of EVs to deliver packaged miRNAs into target cells and to exert biological functions. We applied well-defined approaches to produce and characterize purified EVs with or without specific viral miRNAs. We found that only a small fraction of EVs carried miRNAs. EVs readily bound to different target cell types, but there was no EV-cell membrane fusion or delivery of cargo. Importantly, the functionality of cells exposed to miRNA-carrying EVs was not affected. These results suggest EV-borne miRNAs do not act as effectors and question their relevancy in paracrine cell-to-cell communication.
\end{abstract}

\title{
AUTHOR SUMMARY
}

The majority of metazoan cells release vesicles of different types and origins, such as exosomes and microvesicles, now collectively termed extracellular vesicles (EVs). EVs have gained much attention because they contain microRNAs (miRNAs) and thus could regulate their specific mRNA targets in recipient or acceptor cells that take up EVs. Using a novel fusion assay with superior sensitivity and specificity, we revisited this claim but found no convincing evidence for an efficient functional uptake of EVs in many different cell lines and primary human blood cells. Even EVs engineered to fuse and deliver their miRNA cargo to recipient cells had no measurable effect on target mRNAs in very carefully controlled, quantitative experiments. Our negative results clearly indicate that EVs do not act as vehicles for miRNA-based cell-to-cell communication. 


\section{INTRODUCTION}

Cells release different types of extracellular vesicles (EVs) into the extracellular space. EVs have been reported to transfer proteins and RNA molecules from cell to cell and are thought to be important vehicles of intercellular communication (Simons and Raposo, 2009). They are released by a broad range of cell types and have been found in all body fluids, including blood, urine, and saliva (Merchant et al., 2010; Gallo et al., 2012; Zhou et al., 2012). A class of EVs, termed exosomes, can originate from cytoplasmic multivesicular bodies (MVB), which fuse with the cellular plasma membrane to release a burst of EVs. In addition, single EVs can also directly emerge from the plasma membrane to give rise to microvesicles (Keller et al., 2006; Yáñez-Mó et al., 2015). Exosomes are 40-100 $\mathrm{nm}$ in diameter, and microvesicles can be up to $1000 \mathrm{~nm}$. They have similar biophysical properties and are therefore difficult to study separately (Raposo and Stoorvogel, 2013). In this work, we use the term EVs to include both classes of vesicles.

microRNAs (miRNAs) are small noncoding RNAs 19-22 nt in length, which have important roles in the post-transcriptional regulation of gene expression. miRNAs act intracellularly, but a small fraction are found in the extracellular environment and in different biological fluids in vivo as well as in cell-culture media in vitro (Théry et al., 2006; Sohel, 2016). Extracellular miRNAs are thought to be promising circulating biomarkers for several cancers and other diseases (Turchinovich et al., 2011) as the cancerous cells release typical miRNA species of diagnostic value (Taylor and Gercel-Taylor, 2008; Creemers et al., 2012; Melo et al., 2014).

miRNAs within EVs have been characterized extensively. EVs released from different cell types contain miRNAs and are delivered to other target cells, where the miRNAs regulate their cognate target genes at the posttranscriptional level (Pegtel et al., 2010; Mittelbrunn et al., 2011; Haneklaus et al., 2012; Montecalvo et al., 2012). miRNAs have been considered to be exclusively released within and protected by EVs since circulating miRNAs are extremely stable and resistant to RNases and have been detected in EV preparations purified from many cell types (Wang et al., 2010; Hoy and Buck, 2012). In contrast, two groups independently reported that extracellular miRNAs are rarely contained in EVs but predominantly associated with RNA binding proteins, such as AGO2, that protect extracellular miRNAs from degradation by ubiquitous RNases (Arroyo et al., 2011; Turchinovich et al., 2011; Chevillet et al., 2014). How these EV-free miRNAs are released from cells and whether they are taken up and functional in recipient cells is still uncertain. In addition, an analysis of the stoichiometry of miRNAs contained in exosomes suggested that EVs carry only low numbers of miRNA molecules that are too few to make a biologically significant difference in recipient cells (Chevillet et al., 2014). A deeper knowledge of cell-to-cell transfer of miRNAs is needed to further address this controversy, but it is extremely challenging to characterize the functionality of EV-borne miRNAs in recipient cells because they usually express the very same endogenous miRNAs species. This major problem precludes an accurate evaluation of the transferred miRNAs and their functionality.

Here we used viral miRNAs released from human B cells latently infected with EpsteinBarr virus (EBV) as a model to characterize the role of EV-contained viral miRNAs and their known functions in target cells. In this model, viral miRNAs delivered by EVs are genetically distinct from human miRNAs. Thus, the transferred miRNAs, their uptake and functions in recipient cells can be easily discriminated from host miRNAs. This model has been already employed by others to study the role of EBV's miRNAs. Several groups reported that EVs from latently EBV B cells can deliver viral miRNAs to target cells regulating certain cellular mRNA targets in monocyte-derived dendritic cells or the monocytic cell line THP-1 (Pegtel et al., 2010; Haneklaus et al., 2012; Nanbo et al., 2013). However, these studies did not distinguish between 
effects mediated by miRNAs and those mediated by EVs. Our approach makes use of engineered human B cells, which are infected with mutant EBVs that encode or are devoid of EBV's miRNAs (Seto et al., 2010) yet release EVs that contain the entire spectrum of cellular miRNAs, providing an important reagent and reference for experimental validation.

In our study, we confirmed that latently EBV-infected cells release human as well as viral miRNAs of which only a small fraction co-purified with EVs. Depending on the miRNA species, only $5-11 \%$ of all extracellular miRNAs were found inside EVs. Employing a very sensitive and novel assay, we observed a substantial and perhaps specific binding of EVs to a range of different target cells but failed to detect a fusion between EVs and recipient cells and thus a release of EV cargo into their cytoplasm. Given these findings, not surprisingly, we could not confirm a functional role of EV-borne miRNAs in recipient cells. Our experiments also revealed that singlemolecule copies of three different viral miRNA species are found in 300 to $1.6 \times 10^{4} \mathrm{EVs}$, mainly depending on the miRNAs' abundance in EV-releasing cells. In summary, this work documents that an EV-mediated transfer of their miRNA cargo to all recipient cells tested is functionally irrelevant.

\section{RESULTS}

\section{Extracellular vesicles contain only a minority of extracellular miRNAs}

We used human lymphoblastoid B-cell lines (LCLs) latently infected with EBV as a source of EVs to investigate their miRNA content and functionality. EBV encodes 44 miRNAs (Seto et al., 2010) with known or presumed targets and functions (Albanese et al., 2017; Skalsky, 2017), leading to the release of EVs with cellular as well as viral miRNAs.

First, we validated our method to enrich and purify EVs. To do so, LCLs were cultured for 72 hours in cell-culture medium depleted of bovine EVs contained in fetal calf serum (Supplementary Fig. S1), and cell-derived EVs were isolated from conditioned medium by several steps of differential centrifugation (Fig. 1A). Two low-speed centrifugations removed cells and cellular debris (pre-purification), and then two steps of ultracentrifugation pelleted and concentrated EVs ('miniUC pellet' in Fig. 1A). Resuspended EVs were further purified by floating in discontinuous iodixanol (Optiprep) gradients (Fig. 1A). Finally, EVs were quantitated by nanoparticle tracking analysis (NTA), carefully validated for sensitivity and linearity of analysis (Supplementary Fig. S2). EV-sized particles were found at the top of the gradient in fractions 2 and 3 at densities of around $1.05 \mathrm{~g} / \mathrm{mL}$ (Fig. 1B). The presence of EVs in these fractions was also confirmed by western blot immunodetection with antibodies directed against the human protein TSG101 and the viral protein LMP1 (Fig. 1C), which are both enriched in EVs (Verweij et al., 2011). EVs in fractions 2 and 3 were free of other cellular organelles, such as endoplasmic reticulum (ER), as indicated by the absence of calnexin (Fig. 1C). Representative members of cellular and viral miRNA were contained in fractions 2 and 3, indicating that highly enriched EVs and miRNAs co-purify in these gradients (Fig. 1D). The integrity and the quality of EVs in these two fractions were assessed by electron microscopy (Fig. 1E).

To determine if miRNAs that co-purify with EVs constitute the majority of the extracellular miRNAs released from cells, RNA was extracted from samples obtained from all steps of EV preparation before and after discontinuous flotation density gradient ultracentrifugation (Fig. 2A). Characterization of the RNA molecules using an Agilent Bioanalyzer showed a progressive enrichment of small RNAs and a substantial loss of ribosomal RNAs in the more advanced steps of EV purification (Fig. 2B). For example, the RNA profile contained in the 
'miniUC pellet' indicated a clear enrichment of RNA molecules shorter than $200 \mathrm{nt}$ on average and the depletion of RNAs with lengths corresponding to ribosomal RNAs (Fig. 2B).

RNAs from different steps of purification were subjected to absolute miRNA quantification by TaqMan RT-qPCR analysis as described in Materials and Methods and specified in Supplementary Fig. S3. Prior to RNA extraction and purification, samples were spiked with defined copy numbers of a synthetic miRNA, cel-miR-39 (http://www.mirbase.org/), as an external standard and independent reference to account for variabilities during RNA purification and firststrand cDNA synthesis. Synthetic RNA oligonucleotides with the sequences of the mature miRNAs hsa-miR-16 (human), the viral miRNAs ebv-miR-BART1, ebv-miR-BART3, and ebv-miRBHRF1-2, as well as the reference miRNA cel-miR-39, were used as standards for subsequent absolute miRNA quantification and data normalization.

After ultracentrifugation at $100,000 \mathrm{~g}$ for $120 \mathrm{~min}$, the majority of miRNAs did not sediment but remained in the conditioned medium (CM in Fig. 2C), suggesting that few miRNAs are associated with EVs as reported (Arroyo et al., 2011; Turchinovich et al., 2011). The concentration of the four viral miRNAs in conditioned medium correlated approximately with their intracellular abundance (Fig. 2C,D). The concentration of EV particles as quantified by NTA increased eightfold and more than 70-fold in the 'UC pellet' and the 'miniUC pellet' preparations, respectively, compared with EV concentrations in 'conditioned medium' (Fig. 2E), but most of the miRNA molecules did not co-purify with EVs. Among the four different miRNAs, only a modest enrichment in the order of 1.6 to 2.7-fold was observed, comparing 'conditioned medium' and 'miniUC pellet' preparations (Fig. 2F), which led us to conclude that only a minute fraction of miRNAs is associated with EVs.

To validate this hypothesis, we used size exclusion chromatography (SEC) as a second alternative method of EV isolation (Fig. 3A), which allows separating EVs (Fig. 3B; fractions 79) from free protein (fractions 14-20) as documented by NTA, protein quantification (Fig. 3B), and western blot immunodetection of the viral protein LMP1 (Fig 3C). We quantified the miRNA levels in each of the 20 fractions after SEC. In agreement with our initial findings, only low levels of miRNAs were detected with EVs in fractions 7-9, whereas the majority of miRNAs in fractions 16-18 (Fig. 3D) co-purified with free protein (Fig. 3B,D).

These results documented that only a minor fraction of extracellular miRNAs of both EBV and human origin is associated with EVs.

\section{Binding of extracellular vesicles to target cells suggests specific interactions}

Several groups reported that EVs containing EBV miRNAs are taken up from recipient cells where viral miRNAs regulate the expression of their cognate cellular transcript targets (Pegtel et al., 2010; Haneklaus et al., 2012). To study the functional role of EV-delivered viral miRNAs in target cells, we engineered pairs of LCLs from the same B-cell donors that differ only in the presence (or absence) of viral miRNAs in EVs. Using these pairs of LCLs, we characterized the functions of viral miRNAs that purify with EVs to determine the number of EVs needed to deliver a functional dose of EBV miRNAs to recipient cells.

We sought to identify biologically relevant target cells of EVs released from LCLs. To do so, highly enriched EVs (miniUC pellet) were stained with PKH26, a red fluorescent lipid dye, and they were purified by discontinuous floating density gradient centrifugation to remove unbound, free dye from the EV preparation (Fig. 4A). After purification, we incubated 293T cells with different amounts of PKH26-labeled EVs and corresponding volumes of negative control (PKH26 dye only, purified in parallel). After 4 hours at $37^{\circ} \mathrm{C}, 293 \mathrm{~T}$ cells showed a dose-dependent increase of fluorescent, PKH26-positive cells as quantitated by flow cytometry (Fig. 4B), which was not 
observed when the cells were incubated with the negative control (ctrl, Fig. 4B) or with PKH26 stained EVs at $4{ }^{\circ} \mathrm{C}$ (Fig 4B). We incubated the PKH26-labeled EVs with human PBMCs at $37^{\circ} \mathrm{C}$ for 4 hours and analyzed selected cellular subpopulations by flow cytometry. As reported, monocytes and plasmacytoid DC (pDCs) were intensely stained, whereas human B lymphocytes and dendritic cells showed a lower level of PKH26 staining (Fig. 4C)(Baglio et al., 2016). Interestingly, T cells did not show any staining with PKH26 even when high numbers of EVs were used, suggesting a specific and selective binding of LCL-derived EVs to certain primary cell types and established cell lines (Fig. 4D).

Importantly, PKH26 staining of cells after incubation with dye-labeled EVs indicates a robust cellular interaction and probably also an enrichment of EVs at the level of the cells' plasma membranes. As this method cannot distinguish among binding, internalization, or delivery of EVs and their cargo, we developed a novel functional assay to detect and quantitate EV fusion with or uptake by their putative target cells.

\section{EV-borne delivery of cargo to target cells is inefficient}

To address this important issue, we developed a simple and rapid assay to determine if EVs deliver their content to recipient cells and to quantitate the efficiency of this process. The assay is based on a $\beta$-lactamase reporter $(\mathrm{BlaM})$ molecule and a FRET (Fluorescence or Förster Resonance Energy Transfer) -coupled substrate that can be analyzed by flow cytometry. A lipophilic, esterified form of the substrate (CCF4-AM) can be easily loaded into the cytoplasm of any cell, where it is rapidly converted into its negatively charged form, CCF4, which is retained in the cytosol. CCF4 is very stable, and its $\beta$-lactam ring is only cleaved when $\beta$-lactamase is delivered intact to the cells. The non-cleaved CCF4 substrate and its cleaved derivative can be easily differentiated and quantified by flow cytometry. The BlaM assay has been used extensively with HIV particles to analyze their fusion with and entry into different target cell populations (Cavrois et al., 2014; Jones and Padilla-Parra, 2016).

To study the functions of EVs, we fused a synthetic, codon-optimized version of the BlaM gene to the carboxy-terminus of CD63, a member of the tetraspanin family and cellular receptor enriched in EVs (Escola et al., 1998; Andreu and Yáñez-Mó, 2014 and references therein). We expressed the CD63- $\beta$-lactamase protein (CD63-BlaM) transiently in 293T cells or constitutively in $293 \mathrm{~T}$ cells using a lentiviral vector. EVs harvested from the supernatants of donor cells carried the CD63-BlaM protein, including its intact $\beta$-lactamase activity (Fig. 5A).

As a positive control, we collected EV-containing supernatant from 293T transiently cotransfected with two expression plasmids encoding CD63-BlaM and the vesicular stomatitis virus G (VSV-G). VSV-G is broadly used to pseudo-type retroviral or lentiviral gene vectors because the glycoprotein is incorporated into viral envelopes and ensures a broad tropism and high transduction efficacy of these viral vectors. VSV-G is also incorporated into the membranes of EVs, where it confers membrane fusion with other cells (Meyer et al., 2017 and references therein).

After isolating EVs from CD63-BlaM 293T cells, we incubated recipient 293T cells with EVs for 4 hours. Then the cells were washed, loaded with CCF4-AM, and analyzed by flow cytometry. The CCF4 substrate was readily cleaved in cells incubated with EVs containing CD63BlaM and VSV-G (Fig. 5B, right panel), but no CCF-4 cleavage was detected when cells were incubated with CD63-BlaM-assembled EVs lacking VSV-G or when control cells without EV treatment were analyzed (Fig. 5B, middle and left panels). This experiment indicates that EVs deliver $\beta$-lactamase with high efficiency to target cells, in principle, but only when pseudo-typed with VSV-G. 
We also purified CD63-BlaM-assembled EVs with or without VSV-G and stained both preparations with the dye PKH26 as in Figure 4 to determine if EV surface binding and B-lactamase activity correlate. EVs from both preparations were purified on iodixanol gradients and eight fractions were harvested and analyzed (Fig. 5C). Both EV preparations stained 293T target cells similarly (Fig. 5C, left panel), but only VSV-G-assembled EVs induced cleavage of CCF4 (Fig. $5 \mathrm{C}$, right panel), documenting that EVs efficiently bind to the surface of target cells but do not deliver their cargo into the cytoplasm unless they are equipped with an ectopic fusogenic moiety, such as VSV-G. Our findings suggest that fusion of EVs with cellular membranes of recipient cells with or without receptor-mediated uptake and endocytosis is extremely inefficient (Fig. 5C).

In addition, we assessed the physical number of EV particles by NTA (Supplementary Fig. S2) to titrate purified VSV-G-pseudo-typed and CD63-BlaM assembled EVs using 293T as target cells (Fig. 5D). Within 4 hours, about $1 \times 10^{4}$ physical EV particles per cell were sufficient to transduce half of the cells in this test, indicating a good efficacy and high sensitivity of the fusion assay.

To further validate our findings, other donor and recipient cell combinations were tested for EV-mediated delivery of CD63-BlaM without VSV-G. Since only donor cells need to express the CD63-BlaM reporter to be incorporated into EV membranes, any cell can be used as potential recipient in our EV fusion assay. We engineered 293T, Calu-3, Caco-2, HepG2 and Huh7 cells to express CD63-BlaM constitutively and at high levels using lentiviral transduction techniques. EVs purified from these five different cell lines were incubated with 17 different recipient cells. CD63BlaM assembled EVs lacking VSV-G showed no measurable or extremely low (U-251MG cells) EV uptake (Fig. 5E) and did not spontaneously deliver their cargo to most of the different cell types in PBMCs from several donors (Fig. 5F). Only upon transient co-transfection of 293T cells with VSV-G, EVs contained in the supernatant of these cells readily released their content into recipient cells with different efficacies. Interestingly, recipient cells did not necessarily show the highest uptake when tested with their cognate donor cells (Fig. 5E and Supplementary Fig. S4). Similar results were obtained using LCLs as donor cells (Supplementary Fig S5A).

Next, we tested whether cleavage of the CCF4 substrate could also be detected by fluorescence microscopy. No blue cells were detected in untreated control cells (Supplementary Fig. S5B) but cleaved CCF4 was readily detected in cells incubated with CD63-BlaM containing EVs assembled with VSV-G (Fig. 5G). The blue signal deriving from the CCF-4 cleaved product was homogeneously distributed in the cells' cytoplasm suggesting a direct delivery of CD63-BlaM into this cellular compartment. These observations show that inefficient delivery and transfer of functional cargo contained in the lumen or in the membranes of EVs from six different cell types, including $293 \mathrm{~T}$ and LCLs is due to the lack of an EV-intrinsic fusogenic activity, which nevertheless can be easily introduced by expressing VSV-G during EV biogenesis.

\section{EV-borne miRNAs do not regulate their cognate $3^{\prime}$-untranslated region targets in sensitive reporter assays}

Our previous experiments failed to show a convincing fusion of EVs with membranes of potential recipient cells, but the experiments did not directly determine if miRNAs might still be functionally transferred to target cells by alternative means. To assess this possibility, we used a dual luciferase reporter assay based on psiCHECK2 reporter plasmids equipped with two luciferases, Renilla and firefly. We introduced three copies of perfectly complementary target sites of three different viral miRNAs (ebv-miR-BART1, ebv-miR-BART3, or ebv-miR-BHRF1-2) into the 3' untranslated 
region (UTR) of the Renilla luciferase reporter gene (Fig. 6A). The signal from the firefly luciferase gene was used as an internal control for normalization.

First, we tested the sensitivity of this system by co-transfecting the reporter plasmids with plasmids expressing the three single miRNAs of interest (Fig. 6A and Supplementary Fig. S6B). In general, the miRNA reporter system revealed a very high sensitivity. Transfection of as little as $1.56 \mathrm{ng}$ DNA of a plasmid vector encoding ebv-miR-BHRF1-2 reduced the luciferase activity expressed from the corresponding reporter plasmid by half (Fig. 6A). Fourfold more plasmid DNA was needed with two other expression plasmids encoding ebv-miR-BART1 or ebv-miR-BART3 to reach a similar level of repression (Fig. 6A and Supplementary Fig. S6B). These experiments also suggested that, depending on individual reporter plasmids, 20-300 miRNA copies per cell reduced the luciferase activity by half (data not shown).

Next, we employed $293 \mathrm{~T}$ cells transiently transfected with the three individual miRNA reporter plasmids and added calibrated, increasing doses of purified EVs ('miniUC pellet') harvested from the CM of LCLs as the source of viral miRNAs. As a negative control, we used identically prepared EVs but purified from LCLs infected with a mutant EBV incapable of expressing viral miRNAs ( $\triangle$ miRNA EBV). We incubated the $293 \mathrm{~T}$ reporter cells with up to $1 \times 10^{5}$ EVs per cell for 24 hours but did not observe a specific reduction of Renilla luciferase activity (Supplementary Fig. S6C). (For comparison, 104 VSV-G assembled EVs per cell were sufficient to transduce about $50 \%$ of all cells in Figure 5D.) A very similar result was obtained with THP-1 cells, which modestly bind EVs (Fig. 4D). In this experiment, we used the maximum amount of EVs that the cells tolerated. A higher ratio of EVs per cell led to a reduction of the Renilla luciferase signal probably because a very high EV concentration was toxic to the cells (Supplementary Fig. S7B) as reported (Klinker et al., 2014; Ahmed et al., 2015). In fact, reduction of the Renilla signal was independent of the EV miRNA content (Supplementary Fig. S7A).

\section{Engineered EVs with reporter mRNAs deliver their cargo when assembled with VSV-G, but EV-delivered miRNAs are non-functional in recipient cells}

The experiments so far suggested that either miRNAs levels in EVs are insufficient to regulate their target mRNAs in recipient cells upon EV-mediated delivery or that EV-contained RNA molecules per se are not functional in recipient cells. We addressed this fundamental uncertainty in two experimental settings, shown in panels B and C of Figure 6. In these experiments, 293T cells were used both as donor cells for the generation of EVs and as recipient cells to perform functional analyses upon EV delivery. In the first approach (Fig. 6B), donor cells were transfected with plasmids encoding VSV-G and a dual luciferase reporter as shown in Figure 6A. The intention of this unusual experiment was to generate mRNAs for their EV-mediated delivery and their subsequent evaluation in suitable recipient cells.

VSV-G assembled EVs potentially bearing the mRNAs of the luciferase reporter system were purified. Before incubating the recipient cells, they were transfected with plasmids expressing either ebv-miR-BHRF1-2 or ebv-miR-BART1 to monitor the regulation of transduced reporter mRNAs upon their VSV-G-mediated EV delivery. EVs assembled with VSV-G successfully delivered functional mRNA transcripts encoding the luciferase reporter enzymes to recipient cells (Fig. 6B). Treatment of recipient cells with cycloheximide abrogated miRNA-mediated repression of luciferase activity, showing that the assay discriminates between de novo translated Renilla and firefly luciferases and delivery of luciferase as active enzymes by EV-mediated transfer of proteins. These data demonstrate that the content of EVs is potentially functional when it is delivered into the cytoplasm of recipient cells. It should be noted, however, that these experiments were not 
informative with EVs that had been assembled without VSV-G due to the extremely low signal levels in these trials that were close to background noise of the recording instrument.

In a reciprocal approach (Fig. 6C), donor cells were transfected with plasmids expressing miR-BHRF1-2 or miR-BART1 in combination with VSV-G. EVs generated from the supernatant of transfected cells were purified as before and incubated with recipient cells transfected with very low amounts of the dual luciferase reporter plasmids to monitor the functions of the EV-delivered miR-BHRF1-2 or miR-BART1 miRNAs. In these settings, EVs containing miR-BHRF1-2 or miRBART1 failed to regulate Renilla luciferase activity in recipient cells, although the EVs had been assembled with VSV-G and the luciferase reporter was expressed at very low levels in the recipient cells, only.

These experiments demonstrate that EVs deliver RNA molecules such as mRNAs, in principle, that are then translated to functional protein in recipient cells, but EV-borne transfer of miRNAs is below the detection limit of this assay even when EVs are assembled with VSV-G.

\section{A single miRNA molecule co-purifies with hundreds of EVs}

Since we observed no functional transfer of viral miRNAs even using VSV-G assembled EVs, we decided to calculate the average number of miRNA molecules associated with or contained within a single EV. We isolated and purified EVs from the supernatants of LCLs obtained from different donors from three different sources: the 'miniUC pellet' after two steps of ultracentrifugation (Fig. 2A), the combined fractions 2 and 3 of the discontinuous iodixanol (Optiprep) floating density gradient (Fig. 1A), and the combined fractions 7 and 8 after SEC (Fig. 3D).

Absolute quantification of the different samples revealed that a single specific mature miRNA is found in $300 \mathrm{EVs}$ (in case of the abundant ebv-miR-BHRF1-2) but more often in thousands of EVs (in case of the least abundant ebv-miR-BART1) (Fig. 7). In three differently prepared EV batches, a single EV contained $0.00006 \pm 0.000037,0.00018 \pm 0.00017$ or $0.0034 \pm 0.0018$ copies of ebv-miR-BART1, ebv-miR-BART3 or ebv-miR-BHRF1-2 molecules, respectively. Similar levels were observed with the human miR-16 miRNA $(0.00084 \pm 0.00055$ molecules per EV). Our data are in line with those of Chevillet and colleagues, who quantified human miRNAs contained in highly purified exosomes isolated from conditioned cell culture media or from plasma samples obtained from healthy individuals or patients with ovarian and prostate cancers (Chevillet et al., 2014).

In summary, our results demonstrate that the majority of extracellular miRNAs from cellculture supernatants are not associated with or contained within EVs. The very low copy number of miRNAs found in highly purified EV preparations, together with the very inefficient uptake of EVs, strongly argue against a role of EVs in transferring functionally relevant amounts of miRNAs to recipient cells to modulate their transcriptome or gene expression profiles.

\section{DISCUSSION}

Many groups have reported that miRNAs contained in EVs are involved in a wide range of paracrine and endocrine biological activities and fulfil important functions in different types of target cells (Skog et al., 2008, for a small selection of papers; Mittelbrunn et al., 2011; Hergenreider et al., 2012; Montecalvo et al., 2012; Ramachandran and Palanisamy, 2012; Squadrito et al., 2014). Similarly, viral miRNAs encoded by EBV were reported to be delivered via exosomal transfer from latently infected B cells to recipient cells where viral miRNAs repress their cognate cellular mRNA targets (Pegtel et al., 2010). 
How cellular or viral miRNAs make their way into extracellular vesicles has been elusive. Their random incorporation into EVs appears plausible but active sorting mechanisms have been put forward (Valadi et al., 2007; Skog et al., 2008; Pigati et al., 2010; Mittelbrunn et al., 2011; Guduric-Fuchs et al., 2012; Nolte-'t Hoen et al., 2012; Palma et al., 2012; Dellett et al., 2017), while a very recent publication proposed an interesting LC3 driven process (Leidal et al., 2020). The presence of nuclease-resistant extracellular miRNAs in body fluids and in supernatants of many different cell types cultivated in vitro led to the conclusion that they are contained within the lumen of EVs where they are protected from degradation by ubiquitous RNase activities.

In contrast to this hypothesis, two independent groups found that the majority of circulating miRNAs is mostly complexed with RNA binding proteins such as AGO2 that also prevent degradation by RNases (Arroyo et al., 2011; Turchinovich et al., 2011). The molar concentration of miRNAs found in EVs in our work and the work of others are far below assumed functional levels, which was estimated to be on the order of 100 copies per cell, depending on the abundance of mRNAs target molecules (Brown et al., 2007; Landgraf et al., 2007; Ragan et al., 2011), even when all EVs fused with cell membranes of recipient cells delivering their miRNA cargo (Dragovic et al., 2011; Chevillet et al., 2014; Tosar et al., 2015). Therefore, it appeared to be unlikely that EV-contained miRNAs can modulate target transcript levels in recipient cells.

We revisited this obvious conflicting issue at three functional levels. First, we made use of EBV's biology and engineered LCLs (i.e., human B-cell lines that differ only in their capacity to encode viral miRNAs). Pairs of EV preparations from the same cell type with and without viral miRNAs allow to distinguish between direct effects of miRNAs versus high doses of EVs. We purified EVs released from EBV-infected cells with two different methods, based on their physical density (Figs. 1 and 2) and size (Fig. 3). We analyzed their physical concentration by NTA and quantified the contained miRNAs. In our biochemical approach, less than $5 \%$ of all miRNAs copurified with EVs (Fig. 3), and the majority of miRNAs remained in the supernatant and did not pellet together with EVs (Fig. 2), supporting previous findings (Arroyo et al., 2011; Turchinovich et al., 2011).

Second, we employed a dual luciferase reporter system, consisting of three complementary sequences of the miRNAs of interest downstream of the Renilla luciferase reporter to allow a sensitive detection of the activity of miRNAs (Fig. 6, Supplementary Fig. S6A). Using this approach, we failed to observe viral miRNA activity in recipient cells incubated with EVs, suggesting a failure to transfer functional numbers of miRNAs via EVs in this assay.

Third, we developed a sensitive assay that detects fusion events between EVs and their presumed acceptor cells at a single-cell level (Fig. 5). EVs did fuse with various primary cells contained in PBMCs or established cell lines representing different cell types but only when equipped with a fusogenic protein, such as VSV-G (Fig. 5). The intrinsic capacity of EV to fuse with either plasma membranes of recipient cells or endosomes after receptor-mediated uptake was extremely low (Fig. 5F) or undetectable (Fig. 5C,E) even when using high EV per cell ratios. Only when EVs were assembled with VSV-G, as few as 1000 EVs sufficed to generate a robust specific fusion signal in a single cell (Fig. 5D). Using VSV-G to foster fusion of EVs with cells, we also observed a remarkable delivery of mRNA transcripts coding for two different luciferase reporters (Fig. 6B). However, this approach failed to demonstrate an EV-mediated transfer of functional single miRNA species (Fig. 6C).

EVs from EBV-infected cells physically interact with many cell types (Fig. 4)(Pegtel et al., 2010; Haneklaus et al., 2012; Nanbo et al., 2013). To reevaluate these reports, we stained EVs purified from EBV-infected LCLs with PKH26 dyes. Despite using high numbers of EVs $(>1000 /$ cell), cells of different lineages did not stain uniformly in these experiments, suggesting a 
specific interaction between EVs and certain cell types (Fig. 4C). In fact, even though apparently robust interaction between EVs and various target cells could be observed, an EV-mediated transfer of material was extremely inefficient or was undetectable, according to our newly developed fusion assay (Fig. 5C). Many papers report the successful EV-mediated transfer of miRNAs to different cells regulating intercellular communication as summarized in a recent review (Kalluri and LeBleu, 2020). Our findings strongly argue against a functional transfer of cargo from cell to cell by EVs.

Different methods of preparing EVs might explain this apparent discrepancy. Studying EVs released from different cells is demanding. EVs are small, abundant and probably heterogeneous, and their study requires accurate purification methods and a repertoire of sophisticated instruments, such as nanoparticle tracking analytics, flow cytometry devices among others, as well as standard procedures (EV-TRACK et al., 2017). Still, EVs are difficult to enumerate, and biochemical preparations of EV often contain exogenous vesicles as most cells have to be cultivated with serum components of bovine origin. We took great care to deplete EVs and eliminate protein aggregates from our cell-culture components (Supplementary Fig. S1).

An even more demanding challenge is the analysis of EV-mediated transfer of cargo. More recent approaches include the labeling of EVs with fluorescent dyes or proteins to document protein internalization and luciferase activity in recipient cells in vitro (Tkach and Théry, 2016 for a recent review). A versatile genetic approach makes use of CRE mRNA in the engineered EVs, which is translated upon delivery so that functional CRE protein throws a genetic switch in acceptor cells (Ridder et al., 2014; Zomer et al., 2015). However, free CRE protein is taken up by cells (Peitz et al., 2002) even when it lacks a transduction domain (Will et al., 2002), potentially causing false positive results. A most recent development uses a CRISPR-Cas9-based reporter to document gene editing in recipient cells upon EV-mediated sgRNA transfer (de Jong et al., 2020). By these methods, the transfer of EV content is extremely inefficient (Ridder et al., 2014; Zomer et al., 2015; de Jong et al., 2020). Yet another very recent approach pre-published on bioRxiv used a split luciferase NanoBiT system and demonstrated that a delivery of EV cargo is only detectable, when EVs are assembled with VSV-G (https://doi.org/10.1101/2020.10.16.341974), which is in agreement with our findings reported here.

As some of these techniques seem laborious or prone to false positive results, we developed a novel system that monitors the fusion of EVs with membranes of acceptor cells. This novel 'EV fusion assay' is based on an established method used in the HIV field to study viral entry (Cavrois et al., 2002). In this pioneering approach, $\beta$-lactamase is fused with the viral protein vpr to trap the enzyme in viral infectious particles. Upon infection viral and cellular membranes fuse and $\beta$ lactamase is delivered into the cytoplasmic compartment of HIV-infected cells where it cleaves its substrate (Cavrois et al., 2002; Jones and Padilla-Parra, 2016). We adapted this assay to deliver $\beta$ lactamase via EVs fusing the $\beta$-lactamase gene with CD63, a surface protein that is predominant in EVs. Stable or transient expression of CD63-BlaM in any cell is feasible, non-toxic and readily gives rise to CD63-BlaM-assembled EVs. ß-lactamase as the enzymatic moiety in this setting is advantageous, because only donor cells have to be engineered to deliver CD63-BlaM whereas any class of target cells including primary cells of interest can be tested (Fig. 5E,F). In contrast, other methods including the split luciferase NanoBiT system requires the manipulation of both donor and recipient cells (https://doi.org/10.1101/2020.10.16.341974). The EV fusion assay can be analyzed by flow cytometry or fluorescent microscopy, two easy and fast read-outs. The assay is reliable, quantifiable and free of background noise in combination with flow cytometry (Fig. 5B).

Applying this assay (described in detail in Figure 5A), we learned that EVs equipped with a membrane-targeted (i.e., CD63-fused) B-lactamase protein (Fig. 5E) are taken up by monocytes, $\mathrm{B}$ lymphocytes and pDCs derived from PBMCs but only at a very low rate (Fig. 5F). Only when 
EVs were engineered to contain a virus-derived fusogenic glycoprotein, we found a very efficient transfer of $\beta$-lactamase activity to target cells, indicating that as few as $10^{3}-10^{4} \mathrm{EVs}$ per cell sufficed to detect EV-to-cell fusion events 4 hours post-incubation (Fig. 5D). An incubation period longer than 4 hours did not lead to a marked signal increase (Supplementary Fig. S4B). The EV field is still developing and standardized purification and analytical methods do not yet exist (EVTRACK et al., 2017), but the ß-lactamase fusion assay has the potential to become a routine method for quantitating the uptake of EVs by acceptor cells in an unequivocal, background-free and quantitative manner.

The NTA approach allows the physical quantitation of EVs (Dragovic et al., 2011; Gardiner et al., 2013), which led to the conclusion that the majority of EVs are free of miRNAs (Chevillet et al., 2014). Our findings recapitulate this notion in EV preparations from the supernatants of LCLs (Figs. 3 and 7), arguing that the scarcity of miRNA containing EVs strongly speak against their role as vectors for cell-to-cell communication (Turchinovich et al., 2011; Turchinovich et al., 2016). Moreover, all our attempts to detect a functional miRNA transfer to recipient cells equipped with a highly sensitive detector failed (Fig. 6; Supplementary Fig. S6C). Even when EVs with an engineered fusogenic moiety and a high rate of EV-to-cell transduction were employed, we could not find a convincing functionality of EV-borne miRNAs (Fig. 6C) very much in contrast to EVs engineered to carry functional mRNAs encoding luciferase genes in a reciprocal setting (Fig. 6B).

We designed our experiments to investigate and quantitate the functions of EBV-encoded miRNAs in EVs, but the experiments resulted in a series of negative results as presented in this work. This outcome compares to our published (and unpublished) works with virus particles released from EBV-infected cells that support the productive, lytic phase of this virus (Jochum et al., 2012). EBV particles do contain viral miRNAs at higher numbers than EVs released from latently EBV-infected cells and, very much in contrast to EVs, EBV particles are highly infectious and efficiently target primary immune cells, such as B cells among other cells. Preliminary experiments suggest that viral miRNAs contained in EBV particles can have a direct function in targeted cells where they regulate innate immunity (manuscript in preparation).

In conclusion, our findings clearly demonstrate that the delivery of different species of RNAs as well as proteins through the EVs is an extremely inefficient process. Our data strongly argue against a biologically active transfer of miRNAs as well as of EV cargo in general and at physiologically relevant level, as claimed by others. Whether and to what extent the EVs released from different donor cell types, other than the ones tested here, do carry and specifically release their cargo in a paracrine manner to diverse types of recipient cells needs to be investigated. In this study, we provide different assays that can be easily employed to address these questions in virtually any possible model. 


\section{Materials and Methods}

Cell lines and cell culture. EBV-infected primary human B cells, the resulting lymphoblastoid cell lines (LCLs), peripheral blood mononuclear cell (PBMCs), the monocytic cell lines THP-1, the EBV-positive Burkitt lymphoma cell line Raji and the HEK293-based EBV producer cell lines were maintained in RPMI medium 1640 (Life Technologies). HEK293T cells were maintained in DMEM (Life Technologies). All media were supplemented with $10 \%$ FCS, penicillin $(100 \mathrm{U} / \mathrm{mL}$; Life Technologies), and streptomycin (100 mg/mL; Life Technologies). Cells were cultivated at $37{ }^{\circ} \mathrm{C}$ in a water-saturated atmosphere with $5 \% \mathrm{CO}_{2}$. Cell viability was checked by trypan blue exclusion and cultures with more than $95 \%$ viable cells were used for experiments.

Separation of human primary lymphocytes. Human primary B cells were prepared from adenoidal mononuclear cells by Ficoll Hypaque (PAN Biotech) gradient centrifugation ( as described in Albanese et al., 2016)). B cells were isolated using CD19 MicroBeads and MACS separation columns (Miltenyi Biotec), according to the manufacturer's instruction.

Isolation of extracellular vesicles (EVs). We submitted all relevant data of our experiments to EV-TRACK knowledgebase (EV-TRACK ID: EV200039) (EV-TRACK et al., 2017). EVs were isolated from the supernatant of EBV-infected B cells incubated for 72 hours with medium containing EV-free FCS. We developed the following process to avoid the carry-over of bovine serum-derived EVs to the cell culture medium. FCS was diluted 1:1 with RPMI and centrifuged at $100,000 \mathrm{~g}$ at $4{ }^{\circ} \mathrm{C}$ in a swinging-bucket rotor (SW28 or 32, Beckman Coulter) for $18 \mathrm{~h}$. The supernatant was filter and sterilized using a $0.22-\mu \mathrm{m}$ mesh size filter (Sartorius) and then filtered using a $300 \mathrm{~K}$ Vivaspin 20 (PES, Sartorius) device at $2,000 \mathrm{~g}$ at $10^{\circ} \mathrm{C}$ for $20-30 \mathrm{~min}$. The EV-free FCS was tested for proteins and particle content (Supplementary Fig. S1), aliquoted and stored at $-80{ }^{\circ} \mathrm{C}$.

According to the protocol shown in Figure 1A, EBV-infected B cells were washed with PBS twice and seeded at a density of $0.5 \times 10^{6}$ cells $/ \mathrm{ml}$. After 72 hours, the conditioned cell-culture medium was centrifuged at $300 \mathrm{~g}$ at $20^{\circ} \mathrm{C}$ for $10 \mathrm{~min}$, followed by a second centrifugation at $2,000 \mathrm{~g}$ at $4^{\circ} \mathrm{C}$ for $20 \mathrm{~min}$ to remove cells and debris, respectively.

To generate EVs from $293 \mathrm{~T}$ cells in Figure $6,1 \times 10^{7}$ cells were seeded in a $13-\mathrm{cm}$ dish. The next day, the cells were transfected with $12 \mu \mathrm{g}$ of plasmid DNA encoding one of the two luciferase reporter plasmids or one of the two viral miRNAs (ebv-miR-BART1 or ebv-miR-BHRF1-2) with or without $8 \mu \mathrm{g}$ of a VSV-G expression plasmid as indicated in panels B and C, respectively. At 24 hours after transfection, the medium was replaced with fresh DMEM without FCS. After 72 hours, the conditioned cell-culture medium was collected and processed in the same way as conditioned medium from EBV-infected B cells.

The supernatant was filtered by using a $0.45-\mu \mathrm{m}$ filter (Millipore) and EVs were pelleted by ultracentrifugation (UC) in a $30 \mathrm{ml}$ tube (Kisker Biotech, Cat no UZ-PA-38.5-1) at 100,000 g at $4{ }^{\circ} \mathrm{C}$ for $2 \mathrm{~h}$ in a swinging-bucket rotor (Beckman Coulter; SW28 or SW32).

The supernatant was completely removed and $500 \mu$ of sterile-filtered PBS supplemented with protease inhibitors (Roche, Cat no 11836170001) were added to the bottom of each tube. To resuspend the EVs in the 'UC pellet', the tubes were incubated on ice under agitation for 30 min. The resuspended EVs were then transferred to a 1.5-ml ultracentrifuge tube (Beckman Coulter, Cat no 357448) and centrifuged at $160,000 \mathrm{~g}$ at $4{ }^{\circ} \mathrm{C}$ for $1.5 \mathrm{~h}$ in a fixed-angle rotor to obtain the sediment termed 'miniUC pellet'. The supernatant was completely removed and the miniUC pellet 
was resuspended in filtered PBS. The final volume depended on the subsequent steps. For standard preparation, we started with $180 \mathrm{ml}$ of cell-culture medium to reach a final volume of $1 \mathrm{ml}(180$ fold concentration). For further purification and analysis, $380 \mu 1$ of the miniUC pellet preparation was loaded at the bottom of an iodixanol (Optiprep; Sigma, Cat no D1556) discontinuous density gradient in ultra-clear centrifuge tubes (Beckman Coulter, Cat no 344062). The density gradient was prepared as follows: $380 \mu \mathrm{l}$ of the EV sample was mixed with $520 \mu \mathrm{l}$ of iodixanol (Optiprep) $(60 \%)$ and placed at the bottom of the tube. Then $2.5 \mathrm{ml}$ of a 1:1 dilution of iodixanol and PBS (30\% final concentration of Optiprep) was placed on top to form the middle layer fraction of the gradient. The top layer with a volume of $600 \mu \mathrm{l}$ consisted of filtered PBS to obtain a final total volume of $4 \mathrm{ml}$. The density gradient was centrifuged at $100,000 \mathrm{~g}$ at $4{ }^{\circ} \mathrm{C}$ for $18 \mathrm{~h}$ in a SW60Ti swing-out rotor. In general, 10 fractions $(400 \mu \mathrm{l}$ each) were collected starting from the top. The refractive density of the fractions was measured with a refractometer (Abbe Mark III, Reichert Technologies).

Labelling of EVs with PKH26 membrane dye. EVs were isolated by ultracentrifugation as described above. The EV pellet was resuspended in $200 \mu \mathrm{l}$ of filtered PBS and stained by using the PKH26 Red Fluorescent Cell Linker Kit for General Cell Membrane Labelling (Sigma-Aldrich). The dye solution was freshly prepared by adding $4 \mu \mathrm{l}$ of PKH26 dye to $1 \mathrm{ml}$ of Diluent $\mathrm{C}$. The EV preparation was mixed with $1 \mathrm{ml}$ of Diluent $\mathrm{C}$ before the dye solution was added. The mixture was incubated at room temperature for $5 \mathrm{~min}$ with periodic mixing. Then an equal volume of sterilefiltered $1 \%$ BSA was added and incubated for $1 \mathrm{~min}$ to stop the staining reaction. To wash the sample and reduce its volume, the stained EVs were mixed with PBS and loaded on a 15-ml Amicon Ultra-15 centrifugal filter of $100 \mathrm{~K}$ cutoff (PES; Millipore) and centrifuged at 2,000 $\mathrm{g}$ for 20-30 min at $10^{\circ} \mathrm{C}$ until the volume was reduced to about $400 \mu$ l. Then $380 \mu$ l of stained sample were loaded at a bottom of an iodixanol (Optiprep) discontinuous density gradient. Fractions containing the stained EVs (2 and 3) were collected and washed three times with $10 \mathrm{ml}$ of filtered PBS in a $100 \mathrm{~K}$ Amicon Ultra-15 centrifugal filter at $2000 \mathrm{~g}$ for $10-20 \mathrm{~min}$ at $10{ }^{\circ} \mathrm{C}$. Concentrated stained EVs were resuspended in $1 \mathrm{ml}$ of RPMI containing EV-free FCS.

Nanoparticle tracking analysis. Nanoparticle tracking analysis (NTA) was performed with the ZetaView PMX110 instrument (ParticleMetrix) and the corresponding software (ZetaView 8.04.02) was used to measure the number and the size distribution of the vesicle preparations. Samples were diluted in filtered PBS to achieve a particle concentration in between $1 \times 10^{7}$ to $1 \times 10^{8}$ particles $/ \mathrm{ml}$. Within this range, we confirmed the linearity of data acquisition with beads of known concentration and size $(102.7 \pm 1.3 \mathrm{~nm}$, Polysciences) comparable with EVs (Supplementary Fig. S2). We also determined the range of accuracy of absolute particle quantification by NTA to be in the range of $1 \times 10^{7}$ to $1 \times 10^{8}$ particles $/ \mathrm{ml}$, again using dilutions of calibration beads of known initial particle concentration (Supplementary Fig. S2). All samples were measured at 11 positions with three reading cycles at each position. Pre-acquisition parameters were set to a sensitivity of 75, a shutter speed of 50, a frame rate of 30 frames per second and a trace length of 15 . The postacquisition parameters were set to a minimum brightness of 20, a minimum size of 5 pixels and a maximum size of 1000 pixels.

Size exclusion chromatography (SEC). SEC was performed using qEV columns (Izon science), following the manufacturer's protocol. EBV-infected B cells were washed twice with PBS and 
seeded at a density of $0.5 \times 10^{6}$ cells/ml in RPMI, supplemented with $2 \%$ EV-free FCS, penicillin $(100 \mathrm{U} / \mathrm{mL})$, and streptomycin $(100 \mathrm{mg} / \mathrm{mL})$. After $72 \mathrm{~h}$, the conditioned cell-culture medium was centrifuged at $300 \mathrm{~g}$ for $10 \mathrm{~min}$ at $20^{\circ} \mathrm{C}$, followed by a second centrifugation at 2,000 $\mathrm{g}$ for $20 \mathrm{~min}$ at $4{ }^{\circ} \mathrm{C}$. The supernatant was then concentrated 10 -fold using the $100 \mathrm{~K}$ Amicon Ultra-15 centrifugal filter at $2,000 \mathrm{~g}$ for $10-20 \mathrm{~min}$ at $10{ }^{\circ} \mathrm{C}$. $1 \mathrm{ml}$ of the concentrated supernatant was used for SEC and 20 fractions (500 $\mu 1$ for each) were collected. $800 \mu \mathrm{l}$ of Qiazol (Qiagen) was added to $300 \mu \mathrm{l}$ of each fraction for RNA extraction. $10 \mu \mathrm{l}$ of each fraction were used for western blots (WBs). All parameters of preparing EVs are available at EV-TRACK knowledgebase (http://evtrack.org/review.php) with the EV-TRACK ID EV200039.

Protein extraction and Western blot analysis. Cells were lysed with RIPA buffer [50 mM Tris $\cdot \mathrm{HCl}(\mathrm{pH} 8), 150 \mathrm{mM} \mathrm{NaCl}, 0.1 \% \mathrm{SDS}, 1 \%$ Nonidet P-40, 0.5 \% DOC], kept $30 \mathrm{~min}$ on ice and then stored at $-80^{\circ} \mathrm{C}$. Protein concentrations were quantified with the Pierce BCA protein assay kit (Thermo Scientific), following the manufacturer's protocol. 5-20 $\mu$ l of samples were loaded per well, depending on the source of the proteins. The absorbance was measured at $550 \mathrm{~nm}$ in an EL800 Universal microplate reader (BioTek instruments) For samples from SEC, RIPA buffer was not used and the protein concentration was measured with Bradford assay (Millipore).

Absorbance values of the unknown samples were determined by the interpolation with the BSA standard values using a four-parameter logistic (4PL) curve (Graphpad). Cell lysates or proteins from the different $\mathrm{EV}$ preparations were diluted in Laemmli buffer and denatured at $95{ }^{\circ} \mathrm{C}$ for $5 \mathrm{~min}$. Proteins were separated on 10-12\% SDS- polyacrylamide gel electrophoresis gel (SDSPAGE; Carl Roth) and transferred to nitrocellulose membranes (GE Healthcare Life Science). Membranes were blocked for $1 \mathrm{~h}$ with $5 \%$ non-fat milk in PBS-T (PBS with $0.1 \%$ Tween-20) and incubated with the antibody of interest. Secondary antibodies conjugated with horseradish peroxidase were used and exposed to CEA films (Agfa HealthCare). SDS-PAGE was stained with Coomassie (Serva Blue G-250) for 5 min and destained with a solution of $15 \%$ acetic acid in $\mathrm{H}_{2} \mathrm{O}$. The following antibodies were used for WB: mouse anti-TSG101 1:2000 (4A10; Genetex), mouse anti-Calnexin 1:1000 (610523; BD Bioscience), and rat anti-LMP1 (1G6-3; Helmholtz Zentrum München).

RNA extraction. RNA was isolated using the miRNeasy Kit (Qiagen), according to the manufacturer's protocol. Pellets of cells or EVs were resuspended in $700 \mu 1$ of Qiazol (Qiagen), whereas $800 \mu \mathrm{l}$ of Qiazol were added to 200-300 $\mu$ l of liquid samples, such as cell supernatants or SEC fractions. Samples were vortexed for $10 \mathrm{sec}$, incubated at RT for $5 \mathrm{~min}$, frozen on dry ice, and stored at $-80{ }^{\circ} \mathrm{C}$.

For RNA extraction, samples were thawed on ice. $2 \mu 1$ of RNA-grade glycogen (Thermo scientific) were added to improve RNA recovery. We also added $10 \mu 1\left(10^{7}\right.$ copies $)$ of the synthetic Caenorhabditis elegans miRNA cel-miR-39 (5'-UCACCGGGUGUAAAUCAGCUUG3'; Metabion) as spike-in control RNA since it has no mammalian homologue. 0.2 volumes of chloroform were added to each sample and mixed by vortexing for $10 \mathrm{sec}$. We then followed the manufacturer's protocol, including also the optional step of washing with the RWT buffer. The RNA was resuspended in $30 \mu \mathrm{l}$ of nuclease-free water (Peqlab) in Eppendorf LoBind microcentrifuge tubes.

RNA quantification and quality analysis. RNA was quantified with a NanoDrop 1000 spectrophotometer (Thermo Scientific), the quality of the RNA from cells and EVs was assessed 
with an Agilent 2100 Bioanalyzer (Agilent), according to the manufacturer's protocol (RNA 6000 Pico Kit).

Stem-loop quantitative RT-PCR of microRNAs. Single-stranded cDNA synthesis was performed for each single miRNA of interest using the TaqMan MicroRNA Reverse Transcription kit (Thermo Scientific), following the manufacturer's protocol. For samples with high amount of RNA, such as cell lysate, $200 \mathrm{ng}$ of RNA was used. For samples with low yield of RNA, such as EVs preparations, RNA was diluted 1:3 with nuclease-free water, and $9 \mu$ of this dilution was used for the reverse-transcription (RT). After the RT, the samples were diluted five times with nucleasefree water and $4 \mu \mathrm{l}$ of these dilutions were applied for qPCR using the TaqMan Fast Advanced Master Mix (Thermo Scientific).

The following TaqMan MicroRNA assay were used: ebv-miR-BHRF1-2-3p (197239 mat); ebv-miR-BHRF1-1 (007757); ebv-miR-BART1-5p (197199_mat); ebv-miR-BART3 (004578_mat); hsa-miR-16 (000391); and cel-miR-39 (000200).

For the absolute quantification of mature miRNAs, RNA oligomers corresponding to the mature miRNA sequences of interest were synthesized (Metabion). The quality and quantity of the synthetic RNA molecules were confirmed using an Agilent 2100 Bioanalyzer and a NanoDrop 1000 spectrophotometer (Supplementary Fig. S3), respectively. A dilution of the synthetic RNAs was prepared freshly immediately before each miRNA quantification. RNA oligoes were diluted with nuclease-free water to concentrations ranging from 10 to $10^{8}$ copies per ml. Quantitative RTPCR was performed in parallel and a standard curve of $\mathrm{C}_{\mathrm{T}}$ (threshold cycle) values was calculated using a four-parameter logistic (4PL) fit (Graphpad) for the interpolation of sample $\mathrm{C}_{\mathrm{T}}$ values. Absolute copy numbers of $C$. elegans cel-miR-39 were used as spike-in miRNA to normalize samples.

Luciferase reporter assays. Three tandem repeats of a miRNA binding site (3x PT) for the EBV miRNA of interest were cloned into the downstream the Renilla luciferase $(R l u c)$ in a psiCHECK2 plasmid (Promega). The following primers were used:

miR-BART1-5p Forward

5'-TCGAGCACAGCACGTCACTTCCACTAAGAAATTCACAGCACGTCACTTCCACTAAGAAATTCAC AGCACGTCACTTCCACTAAGAGC-3'

miR-BART1-5p Reverse

5'-GGCCGCTCTTAGTGGAAGTGACGTGCTGTGAATTTCTTAGTGGAAGTGACGTGCTGTGAATTTC TTAGTGGAAGTGACGTGCTGTGC-3'

miR-BART3-3p Forward

5'-TCGAGACACCTGGTGACTAGTGGTGCGAATTACACCTGGTGACTAGTGGTGCGAATTACACCT GGTGACTAGTGGTGCGGC

miR-BART3-3p Reverse 5'-GGCCGCCGCACCACTAGTCACCAGGTGTAATTCGCACCACTAGTCACCAGGTGTAATTCGCAC CACTAGTCACCAGGTGTC miR-BHRF1-2 Forward 5'-TCGAGTCAATTTCTGCCGCAAAAGATAAATTTCAATTTCTGCCGCAAAAGATAAATTTCAATTTC TGCCGCAAAAGATAGC-3' miR-BHRF1-2 Reverse 5'-GGCCGCTATCTTTTGCGGCAGAAATTGAAATTTATCTTTTGGGGCAGAAATTGAAATTTATCTTT 


\section{TGCGGCAGAAATTGAC-3'}

The primers were annealed and ligated with psiCHECK2 digested with XhoI and NotI (NEB). The pCDH vectors harboring single EBV miRNAs were used as described (Tagawa et al., 2016). $293 \mathrm{~T}$ cells were seeded in a 24 -well plate at a density of $1 \times 10^{5}$ cells/well. After $24 \mathrm{~h}$, cells were co-transfected with $30 \mathrm{ng}$ of specific miRNA reporter plasmid (3x PT psiCHECK2) and different amounts of pCDH vector expressing a miRNA of interest (0.39-100 ng). A pCDH empty vector was added to compensate the different amounts of vector used to reach $100 \mathrm{ng}$ in each condition.

Alternatively, extracellular vesicles were used as source of miRNAs. 4 or $24 \mathrm{~h}$ after transfection different amounts of EVs isolated from WT or $\triangle$ miRNA EBV-infected B cells were added. $100 \mu \mathrm{l}$ corresponds to $1 \times 10^{10} \mathrm{EVs}$, if not indicated differently. At $24 \mathrm{~h}$ after DNA transfection, luciferase activities were determined with the Dual-Luciferase Assay Kit (Promega) and the Orion II Microplate Luminometer (Titertek-Berthold). The activity of Rluc was normalized to the activity of Firefly luciferase (Fluc) encoded by the psiCHECK2 plasmid.

MTT assay. EBV-infected B cells were seeded at an optimal concentration of $0.5 \times 10^{6}$ cells $/ \mathrm{ml}$ with different media conditions. After $72 \mathrm{~h}$, viable cells were assessed by an MTT assay (Cory et al., 1991).

CellTiter-Glo. 293T cells or EBV-infected B cells were seeded in a 24-well plate at a density of $1 \times 10^{5}$ cells/well. After $24 \mathrm{~h}$, different amount of EVs isolated from WT EBV-infected B cells were added. At 8 or $24 \mathrm{~h}$ after incubation, cell viability was assessed by CellTiter-Glo 2.0 (Promega), following the manufacturer's protocol. Luminescence signal was measured by the Orion II Microplate Luminometer (Titertek-Berthold).

FACS analysis of PBMCs. Isolated PBMCs were stained with the following antibodies:

CD19 - APC (Clone: HIB19; 17-0199-42, BD Biosciences)

CD3 - APC (Clone: SP34-2; 557597, BD Biosciences)

CD11c - APC (Clone: 3.9; 301613, BioLegend)

CD14 - PE (Clone: MEM-15; 21279144, ImmunoTools)

CD303 - APC (Clone: AC144; 130-097-931, MACS)

CD304 - PE (Clone: REA774; 130-112-045, MACS)

CD123 - VioBlue (Clone: AC145; 130-113-891, MACS)

B-Lactamase (BlaM)-based fusion assay. The expression plasmid p7200 encoding CD63-BlaM was constructed based on pcDNA3.1(+). It encodes the open reading frame of human CD63, which is carboxy-terminally fused (via a $\mathrm{G}_{4} \mathrm{~S}$ flexible linker) to a codon-optimized B-lactamase (BlaM) gene. The CD63-BlaM expression cassette was moved into the context of a lentiviral vector backbone, based on a $\mathrm{pCDH}$ derived vector (System Biosciences) so that mtagBFP, an enhanced monomeric blue fluorescent protein, is co-expressed together with CD63-BlaM. The lentiviral vector was termed p7250.

To generate CD63-BlaM assembled EVs from 293 T cells $1 \times 10^{7}$ cells were seeded in a 13$\mathrm{cm}$ dish. After overnight incubation, $12 \mu \mathrm{g}$ of CD63-BlaM plasmid DNA p7200 was chemically complexed and transfected alone or together with $8 \mu \mathrm{g}$ of the VSV-G expression plasmid p5451. The next day, the medium was exchanged with non-supplemented, plain DMEM cell culture 
medium with $5 \mathrm{~g} / \mathrm{L}$ D-glucose. After $72 \mathrm{~h}$, the supernatant (conditioned medium) was harvested. EVs were isolated by serial centrifugation and density gradient as described above.

To generate CD63-BlaM assembled EVs from stably transduced LCLs and 293T cells, the cells were transduced with the lentiviral vector $\mathrm{p} 7250$, which expresses mtagBFP, an enhanced monomeric blue fluorescent protein, a T2A element, and the open reading frame encompassing CD63-BlaM. Lentivirally transduced cells were enriched by FACS according to their highest expression of mtagBFP. The cells were seeded at an initial density of $5 \times 10^{5} / \mathrm{ml}$ and the conditioned medium was harvested $72 \mathrm{~h}$ later. EVs were isolated by serial centrifugation and density gradient as described above.

The conditioned cell-culture medium or purified EVs from donor cells were incubated with $2 \times 10^{5}$ recipient cells at $37^{\circ} \mathrm{C}$ for $4 \mathrm{~h}$. Cells were washed, trypsinized and collected at $500 \mathrm{~g}$ for 10 min. Cells were resuspended with $100 \mu \mathrm{l}$ of CCF4-AM staining solution in a 96-well plate. The staining solution per $\mathrm{ml}$ consisted of $1 \mathrm{ml}$ of $\mathrm{CO}_{2}$-independent cell-culture medium (Thermo Fisher Scientific, Cat no 18045), $2 \mu 1$ of CCF4-AM, $8 \mu$ of Solution B (Thermo Fisher Scientific, Cat no $\mathrm{K} 1095$ ) and $10 \mu \mathrm{l}$ of $250 \mathrm{mM}$ Probenecid (Sigma, Cat no P8761). Cells were incubated at room temperature in the dark for $16 \mathrm{~h}$. Subsequently, the cells were washed with PBS twice. 293T cells were fixed with $4 \%$ PFA (Merck, Cat no 104005) for $30 \mathrm{~min}$, whereas PBMCs were processed further omitting the fixation step. The measurement was performed by flow cytometry using an LSR Fortessa instrument (BD). The 409-nm wavelength laser (violet) was used for excitation of the FRET substrate, and the emission of the intact, non-cleaved CCF4 substrate was detected at $520 \mathrm{~nm}$ (green), whereas the emission of the cleaved CCF4 substrate was detected at $447 \mathrm{~nm}$ (blue).

EBV production and virus titration with Raji cells. The recombinant EBV genomes used in this study are the plasmid p2089 (Delecluse et al., 1998), a wild-type (WT) EBV, and the p4027, knockout for all the viral miRNAs ( $\triangle$ miR EBV) (Seto et al., 2010).

Virus used to infect primary B cells was produced as described (Seto et al., 2010). Briefly, HEK293-based producer cell lines, which stably carry recombinant EBV genomes, were transfected with plasmids coding for the viral proteins BZLF1 and BALF4 to induce the viral lytic phase. Supernatant was collected 3 days after transfection and titrated using Raji cells as described (Seto et al., 2010; Steinbrück et al., 2015; Pich et al., 2019). Isolated primary B cells were infected with a multiplicity of infection of 0.1 Green Raji Units (GRU). At $18 \mathrm{~h}$ later, the infected B cells were washed and cultivated at an optimal initial density of $5 \times 10^{5}$ cells $/ \mathrm{ml}$.

Confocal Microscopy. The adherent cell lines U-251MG, MDA-MB-231 and LN-18 were seeded on glass coverslips (Carl Roth) coated with fibronectin (Advanced Biomatrix). After 24 hours, medium was replaced and CD63-BlaM assembled EVs with VSV-G purified from 293T were added to the cells. Cells untreated are used in parallel as negative control. Four hours later cells were washed three times with PBS, loaded with CCF4-AM and developed overnight as described above. Subsequently, cells were washed three times with PBS, fixed with $4 \%$ PFA for 10 min at room temperature and washed again. The coverslips were mounted with ProLong ${ }^{\mathrm{TM}}$ Diamond Antifade Mountant (Thermo Fischer Scientific) and were analyzed with a spinning disk confocal microscope (Nikon) using a 60x objective lens. The Imaris software was used to analyze the images. This filter configuration used led to a slight spillover of the cleaved CCF-4 (blue channel) into the CCF-4 (green) channel (see Supplementary Fig. S5B).

Negative stain transmission electron microscopy (TEM). EVs released from LCLs were collected after iodixanol density gradient centrifugation (fractions 2 and 3) and 100K Amicon 
Ultra-15 centrifugal filters were used to transfer the EVs to HEPES-Buffered Saline (HBS). Aliquots of $5 \mathrm{ul}$ of EV preparations in HEPES buffered saline at an approximate concentration of $3.4 \times 10^{13}$ particles per $\mathrm{ml}$ were placed on glow-discharged continuous carbon film supported copper grids ( $3 \mathrm{~mm}, 300 \mathrm{mesh}$, Plano) and adsorbed for $5 \mathrm{~min}$. After sample removal, grids were stained for $30 \mathrm{sec}$ with uranyl acetate $(2 \% \mathrm{w} / \mathrm{v})$. Micrographs were imaged with a 40,000 fold magnification $(0.414 \mathrm{~nm} / \mathrm{pix})$ using a JEOL JEM-1400 Plus microscope operating at $120 \mathrm{kV}$ with a JEOL CCD Ruby camera. The underfocus was set to $500 \mathrm{~nm}$. 


\section{Acknowledgements}

We would like to thank Regina Feederle, Helmholtz Zentrum München, Core Unit Monoclonal Antibodies, German Research Center for Environmental Health, for providing antibodies crucial for this work. We would like to acknowledge Francesca Pinci for critical reading of our manuscript. This work was financially supported by grants from the Deutsche Forschungsgemeinschaft (grant numbers SFB1064/TP A13, SFB-TR36/TP A04), Deutsche Krebshilfe (grant number 70112875), and National Cancer Institute (grant number CA70723) to W.H.

\section{Author contributions}

MA and Y-FAC performed most of the experiments; $\mathrm{CH}, \mathrm{KG}$, and $\mathrm{CG}$ provided additional experimental work; EMP established the CD63-BlaM cell lines; MS and AKS performed EM; MA, Y-FAC, TT, and WH wrote the paper; MA, Y-FAC, TT, RZ, OTK, and WH designed the scientific concept and the experiments.

\section{Competing interests}

The authors declare no competing interests. 


\section{References}

Ahmed, W., P.S. Philip, S. Attoub, and G. Khan. 2015. Epstein-Barr virus-infected cells release Fas ligand in exosomal fractions and induce apoptosis in recipient cells via the extrinsic pathway. J Gen Virol. 96:3646-3659. doi:10.1099/jgv.0.000313.

Albanese, M., T. Tagawa, M. Bouvet, L. Maliqi, D. Lutter, J. Hoser, M. Hastreiter, M. Hayes, B. Sugden, L. Martin, A. Moosmann, and W. Hammerschmidt. 2016. Epstein-Barr virus microRNAs reduce immune surveillance by virus-specific CD8+ T cells. Proc Natl Acad Sci U S A. 113:E6467-E6475. doi:10.1073/pnas.1605884113.

Albanese, M., T. Tagawa, A. Buschle, and W. Hammerschmidt. 2017. microRNAs of EpsteinBarr virus control innate and adaptive anti-viral immunity. J Virol. 91:e01667-16. doi:10.1128/JVI.01667-16.

Andreu, Z., and M. Yáñez-Mó. 2014. Tetraspanins in extracellular vesicle formation and function. Front Immunol. 5:442. doi:10.3389/fimmu.2014.00442.

Arroyo, J.D., J.R. Chevillet, E.M. Kroh, I.K. Ruf, C.C. Pritchard, D.F. Gibson, P.S. Mitchell, C.F. Bennett, E.L. Pogosova-Agadjanyan, D.L. Stirewalt, J.F. Tait, and M. Tewari. 2011. Argonaute 2 complexes carry a population of circulating microRNAs independent of vesicles in human plasma. Proc Natl Acad Sci U S A. 108:5003-5008. doi:10.1073/pnas.1019055108.

Baglio, S.R., M.A. van Eijndhoven, D. Koppers-Lalic, J. Berenguer, S.M. Lougheed, S. Gibbs, N. Léveillé, R.N. Rinkel, E.S. Hopmans, S. Swaminathan, S.A. Verkuijlen, G.L. Scheffer, F.J. van Kuppeveld, T.D. de Gruijl, I.E. Bultink, E.S. Jordanova, M. Hackenberg, S.R. Piersma, J.C. Knol, A.E. Voskuyl, T. Wurdinger, C.R. Jiménez, J.M. Middeldorp, and D.M. Pegtel. 2016. Sensing of latent EBV infection through exosomal transfer of 5'pppRNA. Proc Natl Acad Sci U S A. 113:E587-96. doi:10.1073/pnas.1518130113.

Brown, B.D., B. Gentner, A. Cantore, S. Colleoni, M. Amendola, A. Zingale, A. Baccarini, G. Lazzari, C. Galli, and L. Naldini. 2007. Endogenous microRNA can be broadly exploited to regulate transgene expression according to tissue, lineage and differentiation state. Nat Biotechnol. 25:1457-1467. doi:10.1038/nbt1372.

Cavrois, M., C. De Noronha, and W.C. Greene. 2002. A sensitive and specific enzyme-based assay detecting HIV-1 virion fusion in primary T lymphocytes. Nat Biotechnol. 20:11511154. doi:10.1038/nbt745.

Cavrois, M., J. Neidleman, and W.C. Greene. 2014. HIV-1 Fusion Assay. Bio Protoc. 410.21769/bioprotoc. 1212 .

Chevillet, J.R., Q. Kang, I.K. Ruf, H.A. Briggs, L.N. Vojtech, S.M. Hughes, H.H. Cheng, J.D. Arroyo, E.K. Meredith, E.N. Gallichotte, E.L. Pogosova-Agadjanyan, C. Morrissey, D.L. Stirewalt, F. Hladik, E.Y. Yu, C.S. Higano, and M. Tewari. 2014. Quantitative and stoichiometric analysis of the microRNA content of exosomes. Proc Natl Acad Sci US A. 111:14888-14893. doi:10.1073/pnas.1408301111.

Cory, A.H., T.C. Owen, J.A. Barltrop, and J.G. Cory. 1991. Use of an aqueous soluble tetrazolium/formazan assay for cell growth assays in culture. Cancer Commun. 3:207-212. doi: $10.3727 / 095535491820873191$.

Creemers, E.E., A.J. Tijsen, and Y.M. Pinto. 2012. Circulating microRNAs: novel biomarkers and extracellular communicators in cardiovascular disease. Circ Res. 110:483-495. doi:10.1161/CIRCRESAHA.111.247452. 
de Jong, O.G., D.E. Murphy, I. Mäger, E. Willms, A. Garcia-Guerra, J.J. Gitz-Francois, J. Lefferts, D. Gupta, S.C. Steenbeek, J. van Rheenen, S. El Andaloussi, R.M. Schiffelers, M.J.A. Wood, and P. Vader. 2020. A CRISPR-Cas9-based reporter system for single-cell detection of extracellular vesicle-mediated functional transfer of RNA. Nat Commun. 11:1113. doi:10.1038/s41467-020-14977-8.

Delecluse, H.J., T. Hilsendegen, D. Pich, R. Zeidler, and W. Hammerschmidt. 1998. Propagation and recovery of intact, infectious Epstein-Barr virus from prokaryotic to human cells. Proc. Natl. Acad. Sci. U S A. 95:8245-8250. doi:10.1073/pnas.95.14.8245.

Dellett, M., E.D. Brown, J. Guduric-Fuchs, A. O'Connor, A.W. Stitt, R.J. Medina, and D.A. Simpson. 2017. MicroRNA-containing extracellular vesicles released from endothelial colony-forming cells modulate angiogenesis during ischaemic retinopathy. J Cell Mol Med. 21:3405-3419. doi:10.1111/jcmm.13251.

Dragovic, R.A., C. Gardiner, A.S. Brooks, D.S. Tannetta, D.J. Ferguson, P. Hole, B. Carr, C.W. Redman, A.L. Harris, P.J. Dobson, P. Harrison, and I.L. Sargent. 2011. Sizing and phenotyping of cellular vesicles using Nanoparticle Tracking Analysis. Nanomedicine. 7:780-788. doi:10.1016/j.nano.2011.04.003.

Escola, J.M., M.J. Kleijmeer, W. Stoorvogel, J.M. Griffith, O. Yoshie, and H.J. Geuze. 1998. Selective enrichment of tetraspan proteins on the internal vesicles of multivesicular endosomes and on exosomes secreted by human B-lymphocytes. J Biol Chem. 273:2012120127. doi:10.1074/jbc.273.32.20121.

EV-TRACK, C., J. Van Deun, P. Mestdagh, P. Agostinis, Ö. Akay, S. Anand, J. Anckaert, Z.A. Martinez, T. Baetens, E. Beghein, L. Bertier, G. Berx, J. Boere, S. Boukouris, M. Bremer, D. Buschmann, J.B. Byrd, C. Casert, L. Cheng, A. Cmoch, D. Daveloose, E. De Smedt, S. Demirsoy, V. Depoorter, B. Dhondt, T.A. Driedonks, A. Dudek, A. Elsharawy, I. Floris, A.D. Foers, K. Gärtner, A.D. Garg, E. Geeurickx, J. Gettemans, F. Ghazavi, B. Giebel, T.G. Kormelink, G. Hancock, H. Helsmoortel, A.F. Hill, V. Hyenne, H. Kalra, D. Kim, J. Kowal, S. Kraemer, P. Leidinger, C. Leonelli, Y. Liang, L. Lippens, S. Liu, A. Lo Cicero, S. Martin, S. Mathivanan, P. Mathiyalagan, T. Matusek, G. Milani, M. Monguió-Tortajada, L.M. Mus, D.C. Muth, A. Németh, E.N. Nolte-’t Hoen, L. O’Driscoll, R. Palmulli, M.W. Pfaffl, B. Primdal-Bengtson, E. Romano, Q. Rousseau, S. Sahoo, N. Sampaio, M. Samuel, B. Scicluna, B. Soen, A. Steels, J.V. Swinnen, M. Takatalo, S. Thaminy, C. Théry, J. Tulkens, I. Van Audenhove, S. van der Grein, A. Van Goethem, M.J. van Herwijnen, G. Van Niel, N. Van Roy, A.R. Van Vliet, N. Vandamme, S. Vanhauwaert, G. Vergauwen, F. Verweij, A. Wallaert, M. Wauben, K.W. Witwer, M.I. Zonneveld, O. De Wever, J. Vandesompele, and A. Hendrix. 2017. EV-TRACK: transparent reporting and centralizing knowledge in extracellular vesicle research. Nat Methods. 14:228-232. doi: $10.1038 /$ nmeth.4185.

Gallo, A., M. Tandon, I. Alevizos, and G.G. Illei. 2012. The majority of microRNAs detectable in serum and saliva is concentrated in exosomes. PLoS One. 7:e30679. doi:10.1371/journal.pone.0030679.

Gardiner, C., Y.J. Ferreira, R.A. Dragovic, C.W. Redman, and I.L. Sargent. 2013. Extracellular vesicle sizing and enumeration by nanoparticle tracking analysis. $J$ Extracell Vesicles. 210.3402/jev.v2i0.19671.

Guduric-Fuchs, J., A. O’Connor, B. Camp, C.L. O’Neill, R.J. Medina, and D.A. Simpson. 2012. Selective extracellular vesicle-mediated export of an overlapping set of microRNAs from multiple cell types. BMC Genomics. 13:357. doi:10.1186/1471-2164-13-357. 
Haneklaus, M., M. Gerlic, M. Kurowska-Stolarska, A.-A. Rainey, D. Pich, I.B. McInnes, W. Hammerschmidt, L.A.J. O’Neill, and S.L. Masters. 2012. Cutting Edge: miR-223 and EBV miR-BART15 regulate the NLRP3 inflammasome and IL-1beta production. J Immunol. 189:3795-3799. doi:10.4049/jimmunol.1200312.

Hergenreider, E., S. Heydt, K. Tréguer, T. Boettger, A.J. Horrevoets, A.M. Zeiher, M.P. Scheffer, A.S. Frangakis, X. Yin, M. Mayr, T. Braun, C. Urbich, R.A. Boon, and S. Dimmeler. 2012. Atheroprotective communication between endothelial cells and smooth muscle cells through miRNAs. Nat Cell Biol. 14:249-256. doi:10.1038/ncb2441.

Hoy, A.M., and A.H. Buck. 2012. Extracellular small RNAs: what, where, why. Biochem Soc Trans. 40:886-890. doi:10.1042/BST20120019.

Jochum, S., R. Ruiss, A. Moosmann, W. Hammerschmidt, and R. Zeidler. 2012. RNAs in Epstein-Barr virions control early steps of infection. Proc. Natl. Acad. Sci. U S A. 109:E1396-404. doi:10.1073/pnas.1115906109.

Jones, D.M., and S. Padilla-Parra. 2016. The $\beta$-Lactamase Assay: Harnessing a FRET Biosensor to Analyse Viral Fusion Mechanisms. Sensors (Basel). 1610.3390/s16070950.

Kalluri, R., and V.S. LeBleu. 2020. The biology, function, and biomedical applications of exosomes. Science. 36710.1126/science.aau6977.

Keller, S., M.P. Sanderson, A. Stoeck, and P. Altevogt. 2006. Exosomes: from biogenesis and secretion to biological function. Immunol Lett. 107:102-108. doi:10.1016/j.imlet.2006.09.005.

Klinker, M.W., V. Lizzio, T.J. Reed, D.A. Fox, and S.K. Lundy. 2014. Human B Cell-Derived Lymphoblastoid Cell Lines Constitutively Produce Fas Ligand and Secrete MHCII(+)FasL(+) Killer Exosomes. Front Immunol. 5:144. doi:10.3389/fimmu.2014.00144.

Landgraf, P., M. Rusu, R. Sheridan, A. Sewer, N. Iovino, A. Aravin, S. Pfeffer, A. Rice, A.O. Kamphorst, M. Landthaler, C. Lin, N.D. Socci, L. Hermida, V. Fulci, S. Chiaretti, R. Foa, J. Schliwka, U. Fuchs, A. Novosel, R.U. Muller, B. Schermer, U. Bissels, J. Inman, Q. Phan, M. Chien, D.B. Weir, R. Choksi, G. De Vita, D. Frezzetti, H.I. Trompeter, V. Hornung, G. Teng, G. Hartmann, M. Palkovits, R. Di Lauro, P. Wernet, G. Macino, C.E. Rogler, J.W. Nagle, J. Ju, F.N. Papavasiliou, T. Benzing, P. Lichter, W. Tam, M.J. Brownstein, A. Bosio, A. Borkhardt, J.J. Russo, C. Sander, M. Zavolan, and T. Tuschl. 2007. A mammalian microRNA expression atlas based on small RNA library sequencing. Cell. 129:1401-1414. doi:10.1016/j.cell.2007.04.040.

Leidal, A.M., H.H. Huang, T. Marsh, T. Solvik, D. Zhang, J. Ye, F. Kai, J. Goldsmith, J.Y. Liu, Y.H. Huang, T. Monkkonen, A. Vlahakis, E.J. Huang, H. Goodarzi, L. Yu, A.P. Wiita, and J. Debnath. 2020. The LC3-conjugation machinery specifies the loading of RNA-binding proteins into extracellular vesicles. Nat Cell Biol. 22:187-199. doi:10.1038/s41556-0190450-y.

Melo, S.A., H. Sugimoto, J.T. O’Connell, N. Kato, A. Villanueva, A. Vidal, L. Qiu, E. Vitkin, L.T. Perelman, C.A. Melo, A. Lucci, C. Ivan, G.A. Calin, and R. Kalluri. 2014. Cancer exosomes perform cell-independent microRNA biogenesis and promote tumorigenesis. Cancer Cell. 26:707-721. doi:10.1016/j.ccell.2014.09.005.

Merchant, M.L., D.W. Powell, D.W. Wilkey, T.D. Cummins, J.K. Deegens, I.M. Rood, K.J. McAfee, C. Fleischer, E. Klein, and J.B. Klein. 2010. Microfiltration isolation of human urinary exosomes for characterization by MS. Proteomics Clin Appl. 4:84-96. doi:10.1002/prca.200800093. 
Meyer, C., J. Losacco, Z. Stickney, L. Li, G. Marriott, and B. Lu. 2017. Pseudotyping exosomes for enhanced protein delivery in mammalian cells. Int J Nanomedicine. 12:3153-3170. doi:10.2147/IJN.S133430.

Mittelbrunn, M., C. Gutierrez-Vazquez, C. Villarroya-Beltri, S. Gonzalez, F. Sanchez-Cabo, M.A. Gonzalez, A. Bernad, and F. Sanchez-Madrid. 2011. Unidirectional transfer of microRNA-loaded exosomes from T cells to antigen-presenting cells. Nat Commun. 2:282. doi:10.1038/ncomms1285.

Montecalvo, A., A.T. Larregina, W.J. Shufesky, D.B. Stolz, M.L. Sullivan, J.M. Karlsson, C.J. Baty, G.A. Gibson, G. Erdos, Z. Wang, J. Milosevic, O.A. Tkacheva, S.J. Divito, R. Jordan, J. Lyons-Weiler, S.C. Watkins, and A.E. Morelli. 2012. Mechanism of transfer of functional microRNAs between mouse dendritic cells via exosomes. Blood. 119:756-766. doi:10.1182/blood-2011-02-338004.

Nanbo, A., E. Kawanishi, R. Yoshida, and H. Yoshiyama. 2013. Exosomes derived from EpsteinBarr virus-infected cells are internalized via caveola-dependent endocytosis and promote phenotypic modulation in target cells. $J$ Virol. 87:10334-10347. doi:10.1128/JVI.01310-13.

Nolte-'t Hoen, E.N., H.P. Buermans, M. Waasdorp, W. Stoorvogel, M.H. Wauben, and P.A. 't Hoen. 2012. Deep sequencing of RNA from immune cell-derived vesicles uncovers the selective incorporation of small non-coding RNA biotypes with potential regulatory functions. Nucleic Acids Res. 40:9272-9285. doi:10.1093/nar/gks658.

Palma, J., S.C. Yaddanapudi, L. Pigati, M.A. Havens, S. Jeong, G.A. Weiner, K.M. Weimer, B. Stern, M.L. Hastings, and D.M. Duelli. 2012. MicroRNAs are exported from malignant cells in customized particles. Nucleic Acids Res. 40:9125-9138. doi:10.1093/nar/gks656.

Pegtel, D.M., K. Cosmopoulos, D.A. Thorley-Lawson, M.A. van Eijndhoven, E.S. Hopmans, J.L. Lindenberg, T.D. de Gruijl, T. Wurdinger, and J.M. Middeldorp. 2010. Functional delivery of viral miRNAs via exosomes. Proc Natl Acad Sci U S A. 107:6328-6333. doi:10.1073/pnas.0914843107.

Peitz, M., K. Pfannkuche, K. Rajewsky, and F. Edenhofer. 2002. Ability of the hydrophobic FGF and basic TAT peptides to promote cellular uptake of recombinant Cre recombinase: a tool for efficient genetic engineering of mammalian genomes. Proc Natl Acad Sci US A. 99:4489-94.

Pich, D., P. Mrozek-Gorska, M. Bouvet, A. Sugimoto, E. Akidil, A. Grundhoff, S. Hamperl, P.D. Ling, and W. Hammerschmidt. 2019. First days in the life of naive human B lymphocytes infected with Epstein-Barr virus. MBio. 1010.1128/mBio.01723-19.

Pigati, L., S.C. Yaddanapudi, R. Iyengar, D.J. Kim, S.A. Hearn, D. Danforth, M.L. Hastings, and D.M. Duelli. 2010. Selective release of microRNA species from normal and malignant mammary epithelial cells. PLoS One. 5:e13515. doi:10.1371/journal.pone.0013515.

Ragan, C., M. Zuker, and M.A. Ragan. 2011. Quantitative prediction of miRNA-mRNA interaction based on equilibrium concentrations. PLoS Comput Biol. 7:e1001090. doi:10.1371/journal.pcbi.1001090.

Ramachandran, S., and V. Palanisamy. 2012. Horizontal transfer of RNAs: exosomes as mediators of intercellular communication. Wiley Interdiscip Rev RNA. 3:286-293. doi:10.1002/wrna.115.

Raposo, G., and W. Stoorvogel. 2013. Extracellular vesicles: exosomes, microvesicles, and friends. J Cell Biol. 200:373-383. doi:10.1083/jcb.201211138.

Ridder, K., S. Keller, M. Dams, A.K. Rupp, J. Schlaudraff, D. Del Turco, J. Starmann, J. Macas, D. Karpova, K. Devraj, C. Depboylu, B. Landfried, B. Arnold, K.H. Plate, G. Höglinger, H. Sültmann, P. Altevogt, and S. Momma. 2014. Extracellular vesicle-mediated transfer of 
genetic information between the hematopoietic system and the brain in response to inflammation. PLoS Biol. 12:e1001874. doi:10.1371/journal.pbio.1001874.

Seto, E., A. Moosmann, S. Gromminger, N. Walz, A. Grundhoff, and W. Hammerschmidt. 2010. Micro RNAs of Epstein-Barr virus promote cell cycle progression and prevent apoptosis of primary human B cells. PLoS Pathog. 6:e1001063. doi:10.1371/journal.ppat.1001063.

Simons, M., and G. Raposo. 2009. Exosomes--vesicular carriers for intercellular communication. Curr Opin Cell Biol. 21:575-581. doi:10.1016/j.ceb.2009.03.007.

Skalsky, R.L. 2017. Analysis of Viral and Cellular MicroRNAs in EBV-Infected Cells. Methods Mol Biol. 1532:133-146. doi:10.1007/978-1-4939-6655-4_9.

Skog, J., T. Wurdinger, S. van Rijn, D.H. Meijer, L. Gainche, M. Sena-Esteves, W.T.J. Curry, B.S. Carter, A.M. Krichevsky, and X.O. Breakefield. 2008. Glioblastoma microvesicles transport RNA and proteins that promote tumour growth and provide diagnostic biomarkers. Nat Cell Biol. 10:1470-1476. doi:10.1038/ncb1800.

Sohel, M.H. 2016. Extracellular/Circulating MicroRNAs: Release Mechanisms, Functions and Challenges. Achievements in the Life Sciences. 10:175-186. doi:doi.org/10.1016/j.als.2016.11.007.

Squadrito, M.L., C. Baer, F. Burdet, C. Maderna, G.D. Gilfillan, R. Lyle, M. Ibberson, and M. De Palma. 2014. Endogenous RNAs modulate microRNA sorting to exosomes and transfer to acceptor cells. Cell Rep. 8:1432-1446. doi:10.1016/j.celrep.2014.07.035.

Steinbrück, L., M. Gustems, S. Medele, T.F. Schulz, D. Lutter, and W. Hammerschmidt. 2015. K1 and K15 of Kaposi's sarcoma-associated herpesvirus are partial functional homologues of latent membrane protein 2A of Epstein-Barr virus. J Virol. 89:7248-7261. doi:10.1128/JVI.00839-15.

Tagawa, T., M. Albanese, M. Bouvet, A. Moosmann, J. Mautner, V. Heissmeyer, C. Zielinski, D. Lutter, J. Hoser, M. Hastreiter, M. Hayes, B. Sugden, and W. Hammerschmidt. 2016. Epstein-Barr viral miRNAs inhibit antiviral CD4+ T cell responses targeting IL-12 and peptide processing. J Exp Med. 213:2065-2080. doi:10.1084/jem.20160248.

Taylor, D.D., and C. Gercel-Taylor. 2008. MicroRNA signatures of tumor-derived exosomes as diagnostic biomarkers of ovarian cancer. Gynecol Oncol. 110:13-21. doi:10.1016/j.ygyno.2008.04.033.

Théry, C., S. Amigorena, G. Raposo, and A. Clayton. 2006. Isolation and characterization of exosomes from cell culture supernatants and biological fluids. Curr Protoc Cell Biol. Chapter 3:Unit 3.22. doi:10.1002/0471143030.cb0322s30.

Tkach, M., and C. Théry. 2016. Communication by Extracellular Vesicles: Where We Are and Where We Need to Go. Cell. 164:1226-1232. doi:10.1016/j.cell.2016.01.043.

Tosar, J.P., F. Gámbaro, J. Sanguinetti, B. Bonilla, K.W. Witwer, and A. Cayota. 2015. Assessment of small RNA sorting into different extracellular fractions revealed by highthroughput sequencing of breast cell lines. Nucleic Acids Res. 43:5601-5616. doi:10.1093/nar/gkv432.

Turchinovich, A., A.G. Tonevitsky, and B. Burwinkel. 2016. Extracellular miRNA: A Collision of Two Paradigms. Trends Biochem Sci. 41:883-892. doi:10.1016/j.tibs.2016.08.004.

Turchinovich, A., L. Weiz, A. Langheinz, and B. Burwinkel. 2011. Characterization of extracellular circulating microRNA. Nucleic Acids Res. 39:7223-7233. doi:10.1093/nar/gkr254.

Valadi, H., K. Ekstrom, A. Bossios, M. Sjostrand, J.J. Lee, and J.O. Lotvall. 2007. Exosomemediated transfer of mRNAs and microRNAs is a novel mechanism of genetic exchange between cells. Nat Cell Biol. 9:654-659. doi:10.1038/ncb1596. 
Verweij, F.J., M.A. van Eijndhoven, E.S. Hopmans, T. Vendrig, T. Wurdinger, E. CahirMcFarland, E. Kieff, D. Geerts, R. van der Kant, J. Neefjes, J.M. Middeldorp, and D.M. Pegtel. 2011. LMP1 association with CD63 in endosomes and secretion via exosomes limits constitutive NF-kappaB activation. EMBO J. 30:2115-2129. doi:10.1038/emboj.2011.123.

Wang, K., S. Zhang, J. Weber, D. Baxter, and D.J. Galas. 2010. Export of microRNAs and microRNA-protective protein by mammalian cells. Nucleic Acids Res. 38:7248-7259. doi:10.1093/nar/gkq601.

Will, E., H. Klump, N. Heffner, M. Schwieger, B. Schiedlmeier, W. Ostertag, C. Baum, and C. Stocking. 2002. Unmodified Cre recombinase crosses the membrane. Nucleic Acids Res. 30:e59.

Yáñez-Mó, M., P.R. Siljander, Z. Andreu, A.B. Zavec, F.E. Borràs, E.I. Buzas, K. Buzas, E. Casal, F. Cappello, J. Carvalho, E. Colás, A. Cordeiro-da Silva, S. Fais, J.M. Falcon-Perez, I.M. Ghobrial, B. Giebel, M. Gimona, M. Graner, I. Gursel, M. Gursel, N.H. Heegaard, A. Hendrix, P. Kierulf, K. Kokubun, M. Kosanovic, V. Kralj-Iglic, E.M. Krämer-Albers, S. Laitinen, C. Lässer, T. Lener, E. Ligeti, A. Linē, G. Lipps, A. Llorente, J. Lötvall, M. Manček-Keber, A. Marcilla, M. Mittelbrunn, I. Nazarenko, E.N. Nolte-’t Hoen, T.A. Nyman, L. O’Driscoll, M. Olivan, C. Oliveira, É. Pállinger, H.A. Del Portillo, J. Reventós, M. Rigau, E. Rohde, M. Sammar, F. Sánchez-Madrid, N. Santarém, K. Schallmoser, M.S. Ostenfeld, W. Stoorvogel, R. Stukelj, S.G. Van der Grein, M.H. Vasconcelos, M.H. Wauben, and O. De Wever. 2015. Biological properties of extracellular vesicles and their physiological functions. J Extracell Vesicles. 4:27066. doi:10.3402/jev.v4.27066.

Zhou, Q., M. Li, X. Wang, Q. Li, T. Wang, Q. Zhu, X. Zhou, X. Wang, X. Gao, and X. Li. 2012. Immune-related microRNAs are abundant in breast milk exosomes. Int J Biol Sci. 8:118123

Zomer, A., C. Maynard, F.J. Verweij, A. Kamermans, R. Schäfer, E. Beerling, R.M. Schiffelers, E. de Wit, J. Berenguer, S.I.J. Ellenbroek, T. Wurdinger, D.M. Pegtel, and J. van Rheenen. 2015. In Vivo imaging reveals extracellular vesicle-mediated phenocopying of metastatic behavior. Cell. 161:1046-1057. doi:10.1016/j.cell.2015.04.042. 


\section{Figure Legends}

Fig. 1 Isolation and characterization of extracellular vesicles from EBV-infected cells. (A) Schematic overview of our method of extracellular vesicles (EVs) enrichment and purification. EBV-infected B cells, lymphoblastoid cell lines (LCLs), were seeded at an initial density of $0.5 \times 10^{6}$ cells $/ \mathrm{ml}$ in cell-culture medium containing $2 \%$ of EV-depleted fetal calf serum (see Materials and Methods) and processed as indicated. (B) The concentrations of EVs in the 10 fractions after iodixanol (Optiprep) floating density gradient centrifugation were measured by nanoparticle tracking analysis (NTA). One representative preparation is shown. (C) Western blot immunodetection of the EV marker protein TSG101 and the EBV protein LMP1, which are enriched in EVs, and Calnexin as a negative control in the 10 fractions. $5 \mu \mathrm{g}$ of the cell lysate (CL) or resuspended 'miniUC pellet' (mini UC) preparations as indicated in panel A were used as controls. Per density gradient fraction 10,20 , or $60 \mu 1$ were loaded onto the gels to detect LMP1, TSG101, or Calnexin, respectively. One representative preparation of three is shown. (D) Relative levels of four selected miRNAs (three viral miRNAs and a representative human miRNA) were analyzed by TaqMan RT-qPCR analysis to determine their physical density characteristics after floating density ultracentrifugation. All miRNAs are found in fractions 2-4. One representative quantification of three is shown. (E) Electron microscopic analysis of negative-stained EVs after iodixanol density gradient purification. Scale bars is $200 \mathrm{~nm}$.

Fig. 2 microRNAs quantitation during different steps of EV purification. (A) Schematic overview of EV sample collection and purification. After $72 \mathrm{~h}$ of LCL cell culture, we collected (i) the cell supernatant ('conditioned medium'; $180 \mathrm{ml}$ ), (ii) the pellet after ultracentrifugation ('UC pellet'; $9 \mathrm{ml}$ ), (iii) the supernatant after ultracentrifugation ('conditioned medium EV depleted' (180 ml), (iv) the pellet after the second ultracentrifugation step ('miniUC pellet'; $1 \mathrm{ml}$ ), and (v) the remaining supernatant ('UC EV depleted'; $9 \mathrm{ml}$ ). RNA was extracted from $200 \mu \mathrm{l}$ of each sample. As a control 'total RNA' from $1 \times 10^{6} \mathrm{LCLs}$ was prepared. (B) Electropherograms after Bioanalyzer analysis of four samples are shown with their extinction profiles. (C) Using a Taqman stem loop RT-qPCR protocol, absolute copy number of four miRNAs were determined in different steps of EV purification as shown in panel A. For each of the four individual miRNA, a regression function with a synthetic miRNA oligonucleotide was generated by RT-qPCR for its absolute quantification. The different sample volumes were considered, according to the legend of panel A. (D) Absolute copy numbers of a human and three viral miRNAs in $10^{6} \mathrm{EBV}$-infected $\mathrm{B}$ cells (LCLs) are provided. (E) Concentrations of EV particles contained in different samples as indicated were measured by NTA. Numbers indicate fold-changes between 'conditioned medium' and 'UC pellet' $(\times 8)$ and 'conditioned medium' and 'miniUC pellet' $(\times 71.5)$ as explained in panel A. (F) Concentrations of four individual miRNAs (three viral miRNAs and a representative cellular miRNA) are shown in the different steps of EV purification as illustrated in panel A. Error bars in panels $\mathrm{C}-\mathrm{F}$ indicate mean and SD of triplicates. Data obtained from one experiment of two independent experiments are shown.

Fig. 3 The majority of miRNAs does not co-purify with extracellular vesicles. (A) Schematic overview of the purification of EVs by size-exclusion chromatography (SEC). EBV-infected B cells (LCLs) were seeded at an initial density of $0.5 \times 10^{6}$ cells $/ \mathrm{ml}$ in medium containing $2 \%$ of EVfree FCS (see Materials and Methods). After $72 \mathrm{~h}$, the supernatant was harvested and different steps of centrifugation were used to remove cells (300 g for $10 \mathrm{~min}$ ) and cell debris (2000 g for $20 \mathrm{~min})$, 
followed by filtration through a $0.45-\mu \mathrm{m}$ mesh size filter. The conditioned cell-culture medium was further concentrated 10 -fold to a final volume of $1 \mathrm{ml}$ using a $100-\mathrm{kDa}$ centrifugal ultrafiltration device (Amicon). The concentrated conditioned medium was then loaded onto a size-exclusion chromatography qEV column (Izon Science Ltd). 20 fractions of $500 \mu$ each were collected. (B) Concentration of EV particles and protein was measured in each fraction by NTA and a colorimetric Bradford assay, respectively. (C) EVs were found in fractions 7, 8, and 9 as confirmed by Western blot immunodetection with an LMP1-specific antibody. LCLs cell lysate (CL) was used as positive control. The viral LMP1 protein is highly enriched in the membranes of EVs. A non-specific band of about $70 \mathrm{kDa}$ observed in EV-free, protein-enriched fractions 14-20 likely stems from immunoglobulin heavy chain molecules produced by EBV-infected B cells. (D) Using a TaqMan stem loop RT-qPCR, absolute copy numbers of miRNAs were determined in each fraction after size-exclusion chromatography. For each miRNA, a standard regression obtained with a corresponding synthetic miRNA oligonucleotide was used for absolute miRNA quantification. The results show one representative experiment of three.

Fig. 4 EVs from EBV-infected B cells interact preferentially with certain cell types. (A) Schematic overview of the labeling and purification of EVs, which were isolated as described in Figure 1A. Resuspended EVs contained in the 'miniUC pellet' were stained with the dye PKH26 as described in Materials and Methods. After staining, the EVs were concentrated using a 100-kDa Amicon centrifugal filter and then loaded at the bottom of an iodixanol (Optiprep) floating density gradient. After $16 \mathrm{~h}$ at 100,000 g, fractions 2 and 3 containing the EVs were pooled and washed three times with PBS using a 100-kDa Amicon centrifugal filter unit to remove unbound dye. Concentrated and PHK26-stained EVs were finally resuspended and used in the assays shown in panels B and C. A negative control without EVs was mock stained with the same amount of dye and purified in parallel. (B) $293 \mathrm{~T}$ cells were incubated with increasing volumes of PKH26-stained EVs or the negative control (neg. ctrl.) at $37{ }^{\circ} \mathrm{C}$. As control, 293T cells were incubated with the same volume of stained EVs at $4{ }^{\circ} \mathrm{C}$. After $4 \mathrm{~h}, 293 \mathrm{~T}$ cells were analyzed by flow cytometry. The percentage of $\mathrm{PKH} 26$ positive cells is shown as a function of EV volume dose. (C) Peripheral blood mononuclear cells (PBMCs) were incubated with $100 \mu \mathrm{l}$ of PHK26-stained EVs (about 1,000 EVs per cell). After $4 \mathrm{~h}$, PBMCs were stained with antibodies specific for different cell types and analyzed by flow cytometry as indicated above each histogram. A representative example of two independent experiments is shown for each panel. (D) EBV-infected B cells (LCL), THP-1, or $293 \mathrm{~T}$ cells were incubated as described in panel B at $37^{\circ} \mathrm{C}$ for $4 \mathrm{~h}$ and analyzed for the fraction of PKH26-positive cells. The results show one representative experiment of three.

Fig. 5 EVs do not deliver their cargo to recipient cells unless EVs carry a fusogenic glycoprotein. The CD63-conjugated $\beta$-lactamase (CD63-BlaM) fusion protein serves as a reporter to investigate the uptake of EVs by recipient cells. (A) The flow chart depicts the fusion assay starting with conditioned medium (supernatant) or purified preparations of CD63-BlaM containing EVs from donor cells that express the $\beta$-lactamase fusion protein. The target cells were incubated for $4 \mathrm{~h}$, loaded with CCF4-AM substrate overnight, fixed depending on the cell type, and analyzed by flow cytometry. Fixation (in parenthesis) is an optional step and not essential for the assay to work. (The graphics was created with BioRender.com.) (B) $2 \times 10^{5} 293 \mathrm{~T}$ recipient cells were incubated for 4 hours with $50 \mu \mathrm{l}$ of concentrated EVs (about 50,000 EVs per cell) isolated from 293 T donor cells transiently transfected with two expression plasmids encoding CD63-BlaM and VSV-G or with a single plasmid encoding CD63-BlaM, only. After loading the CCF4-AM substrate, 293T recipient cells were analyzed by flow cytometry. CCF4 was readily cleaved in cells 
incubated with CD63-BlaM assembled EVs equipped with VSV-G (right panel), but not in CCF4loaded cells that had been incubated with EVs assembled with CD63-BlaM, only (middle panel) or untreated cells (left panel). (C) 293T donor cells were transfected with an expression plasmid encoding CD63-BlaM alone or together with a second plasmid encoding VSV-G as in panel B. Both EV-containing supernatants were harvested, purified, and the 'UC pellet' was stained with PKH26 as in Figure 4A. Identical volume samples $(50 \mu 1)$ of the eight fractions obtained after iodixanol (Optiprep) density gradient centrifugation were incubated with $2 \times 10^{5} 293 \mathrm{~T}$ recipient cells and analyzed for PKH26 fluorescence and CCF4 cleavage by flow cytometry. Both types of EVs contained in fractions 2-5 of the gradients bound equally well to 293T cells (left panel) as indicated by the percentage of PKH26-positive cells. BlaM-positive cells were only detected when incubated with VSV-G assembled EVs contained in fractions 2-5 but not in cells incubated with VSV-G negative EVs (right panel). (D) 293 T cells were transiently transfected with two expression plasmids encoding CD63-BlaM and VSV-G and the conditioned medium (CM) was collected. It was concentrated (UC pellet as in Figure 4A) and purified by iodixanol (Optiprep) density gradient centrifugation. The concentrations of EVs in conditioned medium $(\mathrm{CM})$, the resuspended UC pellet and fraction 2 of the gradient were analyzed by NTA. $2 \times 10^{5} 293 \mathrm{~T}$ target cells were incubated for $4 \mathrm{~h}$ with increasing amounts of the three EV preparations and the percentage of BlaM-positive cells was determined and plotted versus the number of EV particles used per cell. (E) Heatmap of a set of EV fusion assays with five donor cells and 17 different recipient cells. 293T, Calu-3, Caco-2, HepG2 and Huh7 were engineered to express CD63-BlaM stably after lentiviral transduction. Where indicated, the cells were transiently transfected with an expression plasmid encoding VSVG. $50 \mu \mathrm{l}$ of EVs from donor cells were purified and incubated with $2 \times 10^{5}$ recipient cells from 17 different cell lines for $4 \mathrm{~h}$. The cells were loaded with CCF4-AM substrate, fixed and analyzed by flow cytometry. Mean of three replicates is shown. All single datasets are shown in Supplementary Figure S4A. The concentration of EVs used is shown in Supplementary Figure S4C. (F) PBMCs were incubated with EVs obtained from conditioned medium of $293 \mathrm{~T}$ cells transiently transfected with the CD63-BlaM encoding plasmid, only, or together with an expression plasmid encoding VSV-G. PBMCs were incubated for $4 \mathrm{~h}$, loaded with CCF4-AM substrate, and stained with antibodies to distinguish different cell types contained in PBMCs. The cells were analyzed by flow cytometry and gated for their identity (T cells, B cells, monocytes, DCs, pDCs) and percentages of CCF4 cleavage. Mean and SD of three independent donors are shown. Asterisks indicate statistical significance by paired two-tailed t test. ( $* \mathrm{P} \leq 0.05 ; * * * \mathrm{P} \leq 0.01$; $* * * \mathrm{P} \leq 0.001)$. (G) $\mathrm{U}-251 \mathrm{MG}$ and MDA-MB-231 cells were used as recipient cells and incubated with EVs from 293T cells transfected with plasmids encoding CD63-BlaM and VSV-G as in panel E. The cells were analyzed by confocal microscopy. Scale bars is $30 \mu \mathrm{m}$. Controls are provided in Supplementary Figure S5B.

Fig. 6 Dual luciferase reporter assays indicate a functional EV-mediated transfer of mRNA transcripts but fail to detect miRNAs-dependent regulation of reporter transcripts in recipient cells. (A) The design of the modified dual luciferase reporter plasmid, based on psiCHECK2, is shown, which encompasses the internal control firefly luciferase (used for normalization) and the reporter Renilla luciferase with three tandem copies of perfect complementary target sites (3xPT) of the miRNAs of interest inserted in the 3'UTR of the Renilla mRNA. 293T cells were transfected with $30 \mathrm{ng}$ of the miRNA reporter plasmid containing $3 \times \mathrm{PT}$ with increasing amounts of the corresponding miRNA expression vector ( $\mathrm{pCDH}$ ) starting with 390 pg up to $100 \mathrm{ng}$. At $24 \mathrm{~h}$ after transfection cells were lysed to determine the Renilla and firefly luciferase activities. Mean of three replicates is shown. (B) Reciprocal dual luciferase assays with EVs engineered to transfer luciferase-encoding mRNAs to recipient 293T cells expressing viral 
miRNAs. Left panel: overview of the principal components of the dual luciferase assay. 293T donor cells seeded in a $13-\mathrm{cm}$ dish were transiently transfected with $12 \mu \mathrm{g}$ of $3 \mathrm{x}$ PT psiCHECK2 dual luciferase reporter plasmid DNA in combination with $8 \mu \mathrm{g}$ of a VSV-G expression plasmid. $50 \mu \mathrm{l}$ of purified EVs were transferred to recipient $293 \mathrm{~T}$ cells in 24-well plates transiently transfected with $500 \mathrm{ng}$ expression plasmid DNA encoding miR-BHRF1-2 or miR-BART1 as indicated. For control, the recipient cells were incubated with cycloheximide (CHX; $20 \mu \mathrm{g} / \mathrm{ml})$ to abrogate translation. Middle and right panels: results of reporter assays lysates from recipient 293T cells expressing miR-BHRF1-2 or miR-BART1 as indicated. The transduced mRNAs encoding Renilla luciferase with the perfect complementary target sites (3xPT) for miR-BHRF1-2 or miRBART1 (and firefly luciferase used for normalization) are translated and expressed in the recipient cells, but repressed in cells that contain the matching miRNA. Mean and SD of three independent donors are shown. Asterisks indicate statistical significance by paired two-tailed t test. $(* \mathrm{P} \leq 0.05$; $* * * \mathrm{P} \leq 0.01 ; * * * \mathrm{P} \leq 0.001)$. (C) Dual luciferase assays with reporter constructs $(3 \mathrm{x} \mathrm{PT}$ psiCHECK2) shown in panel A were performed to investigate the functional transfer of EV-borne viral miRNAs to $293 \mathrm{~T}$ recipient cells. Left panel: overview of the dual luciferase assay. 293T cells seeded in a $13-\mathrm{cm}$ dish were transiently transfected with expression plasmids (12 $\mu \mathrm{g})$ coding for miR-BHRF1-2 or miR-BART1 alone or in combination with an expression plasmid coding for VSV-G $(8 \mu \mathrm{g})$. Medium was replaced with fresh medium after 24 hours and the cells were incubated for another $72 \mathrm{~h}$ prior to harvest. $50 \mu \mathrm{l}$ of EVs were transferred to 293T cells in a 24wells plate transiently transfected with $10 \mathrm{ng}$ of dual reporter 3x PT psiCHECK2 plasmid DNA as indicated. Middle and right panels: results of reporter assays show no repression of the luciferase reporters. Ctrl: conditioned medium from 293T donor cells transiently transfected with an expression plasmid encoding no miRNA; VSV-G: conditioned medium from 293T donor cells transiently transfected with a VSV-G encoding expression plasmid, only; Pos Ctrl: recipient 293T cells transiently co-transfected with both the miRNA expression plasmid and the corresponding $3 \mathrm{x}$ PT psiCHECK2 reporter plasmid. Mean and SD of three independent donors are shown. Asterisks indicate statistical significance by paired two-tailed t test. $(* \mathrm{P} \leq 0.05 ; * * * \mathrm{P} \leq 0.01 ; * * * \mathrm{P} \leq 0.001)$.

Fig. 7 Single miRNAs are very scarce in EVs. Three different preparations of EVs were obtained from conditioned medium of LCLs infected with wild-type EBV. EVs were purified by differential centrifugation ('miniUC pellet'; Fig. 2A), by size exclusion chromatography (SEC, fractions 7-9 were combined and analyzed; Fig. 3A), or by iodixanol (Optiprep) density gradient centrifugation (fractions 2 and 3 were combined and analyzed; Fig. 1D). Concentrations of EVs were determined by NTA. RNA was isolated from the three preparations (resuspended miniUC pellet, SEC and density gradient fractions), and absolute copy numbers of four different miRNAs were determined using a TaqMan stem loop RT-qPCR. For each miRNA, a standard curve of a synthetic RNA oligonucleotide (mimicking the miRNA) was established to determine absolute miRNA copy numbers in the preparations. (A) The graph shows the miRNA copy number per EV of three viral miRNAs and the human miRNA hsa-miR-16. (B) The graph indicates the miRNA copy numbers contained in $10^{6}$ EVs (y-axis) as measured by RT-qPCR and NTA, respectively, versus the miRNA copy number contained in $10^{6}$ cells ( $\mathrm{x}$-axis). 


\section{A}

LCL - EBV infected $B$ cells

$0.5 \times 10^{6} \mathrm{cell} / \mathrm{ml}$

72 hours

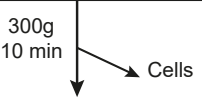

Supernatant

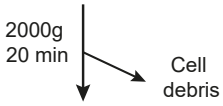

Supernatant

$0.45 \mu \mathrm{m}$

filtration

Conditioned medium (CM)

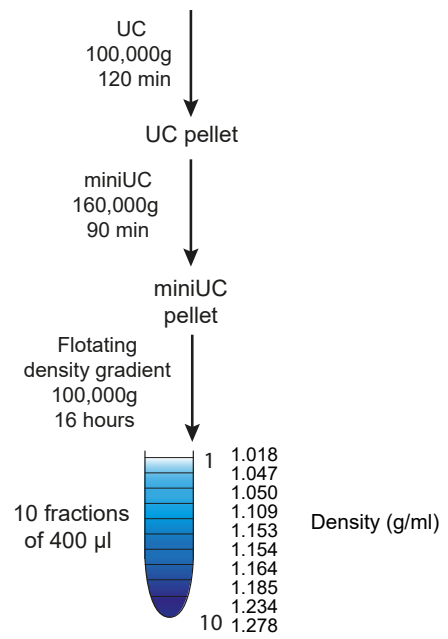

D

$\frac{2}{2}$

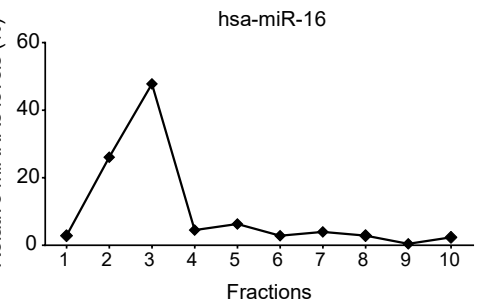

,

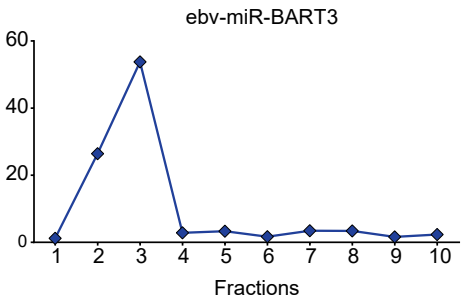

B

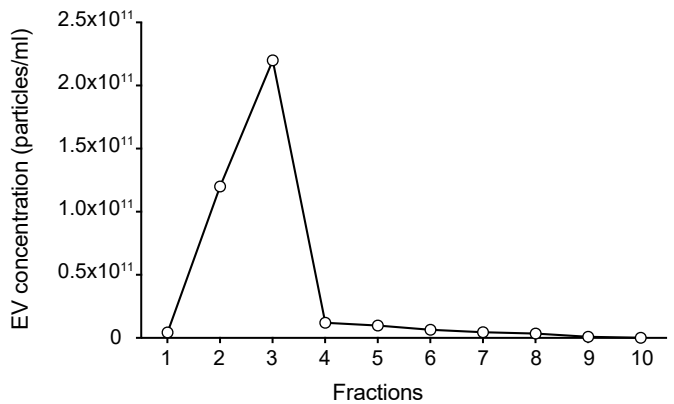

C

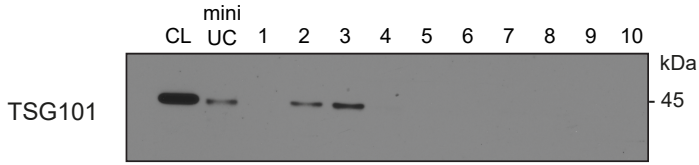

LMP1

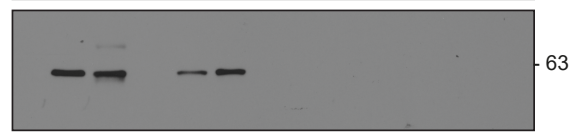

Calnexin

(high exposure)

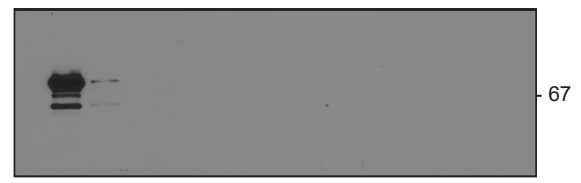

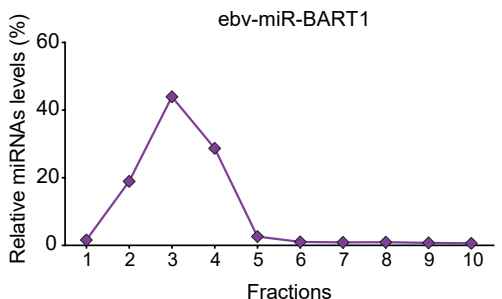

ebv-miR-BHRF1-2

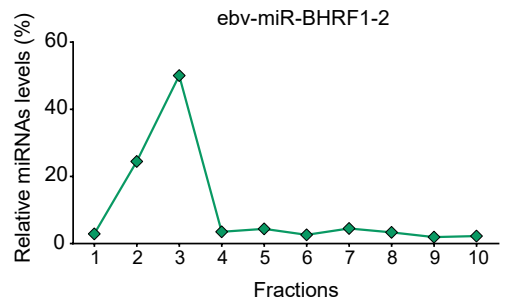

E

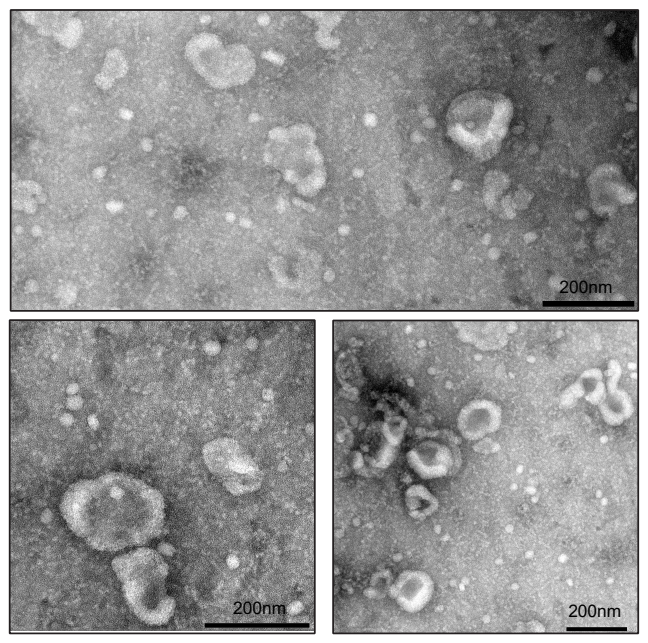

Figure 1 


\section{A}

LCL - EBV infected B cells

$0.5 \times 10^{6} \mathrm{cell} / \mathrm{ml}$

72 hours

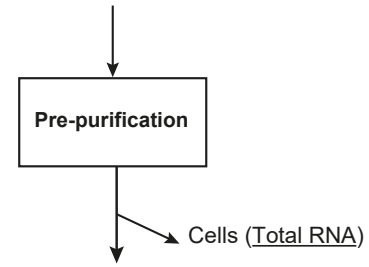

Conditioned medium (CM)

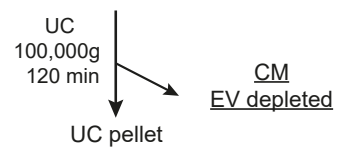

miniUC

$160,000 \mathrm{~g}$

$90 \mathrm{~min}$

$\downarrow$ EV depleted

$\frac{\text { miniUC }}{\text { pellet }}$

C
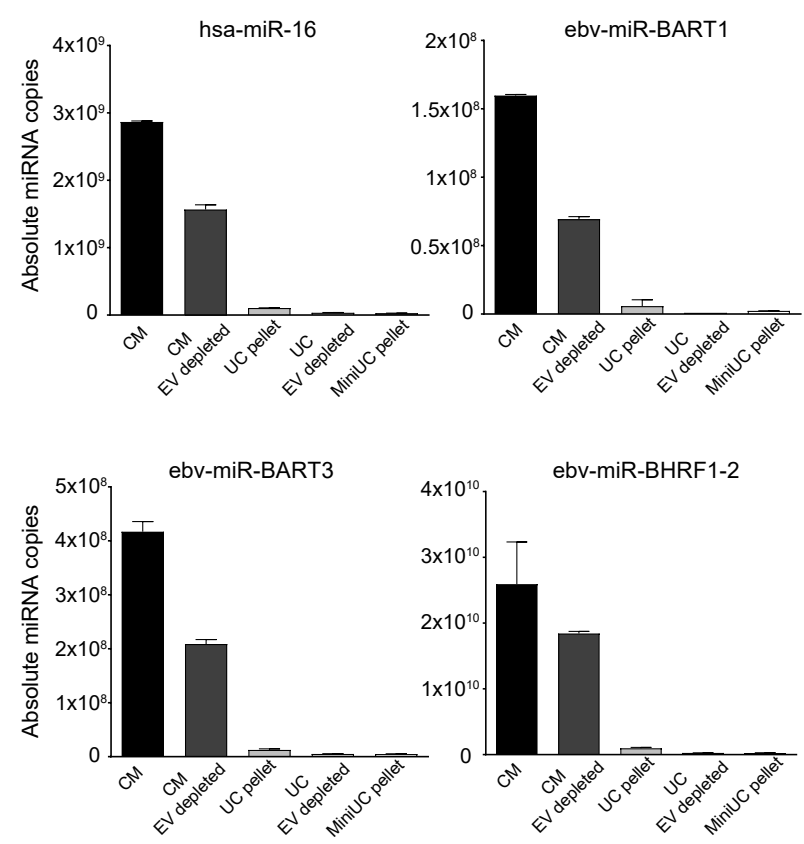

D

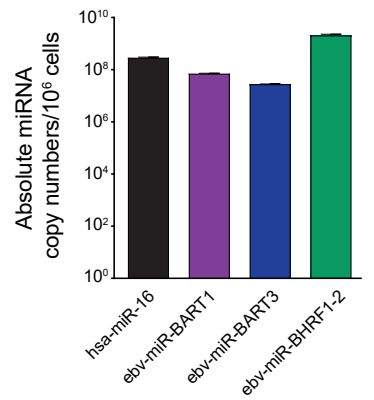

E

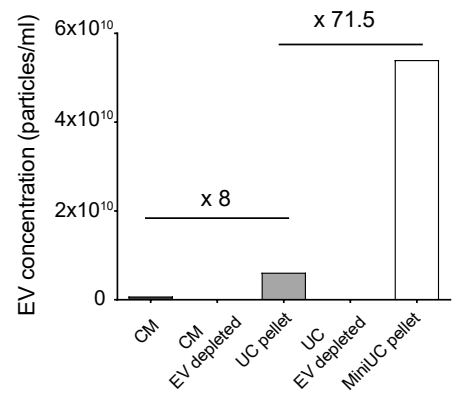

F

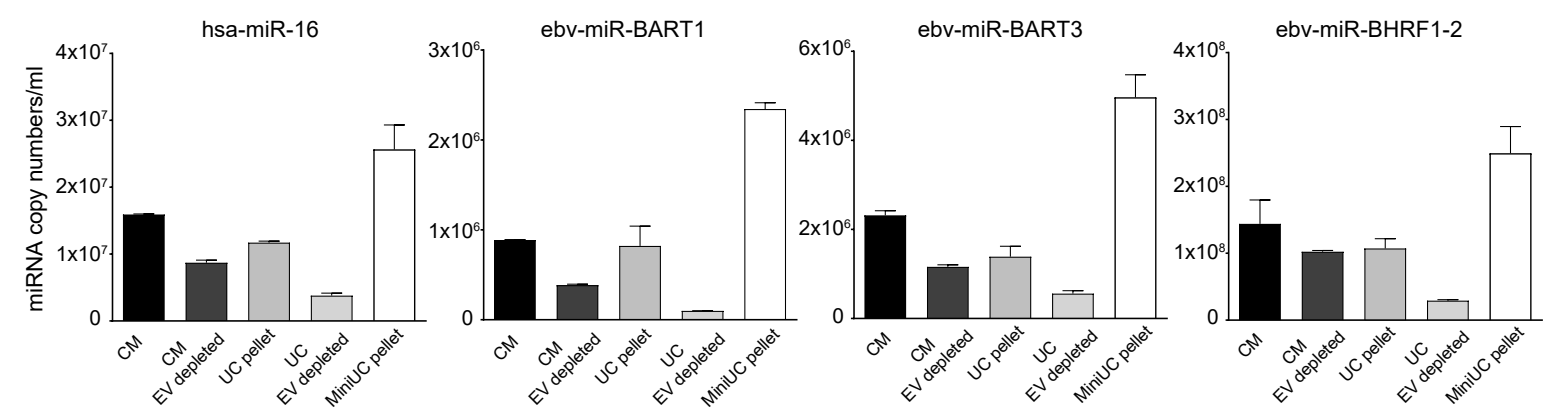

Figure 2 
A

$$
\begin{gathered}
\text { EBV infected B cells } \\
0.5 \times 10^{6} \mathrm{cell} / \mathrm{ml}
\end{gathered}
$$

72 hours

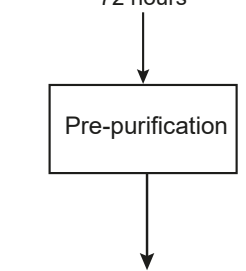

Conditioned medium (CM)

Ultrafiltration
$100 \mathrm{kDa}$ conditioned medium 10x

Size exclusion chromatography

$$
\begin{gathered}
20 \text { fractions } \\
\text { of } 500 \mu \mathrm{l}
\end{gathered}
$$

B

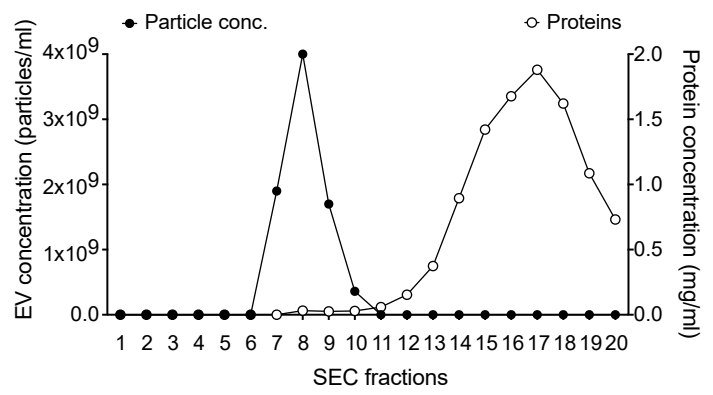

C

LMP1

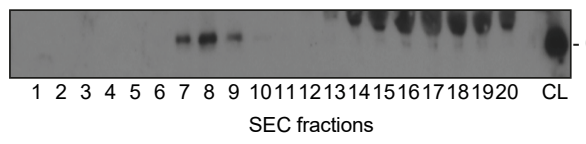

$-63 \mathrm{kDa}$

\section{D}
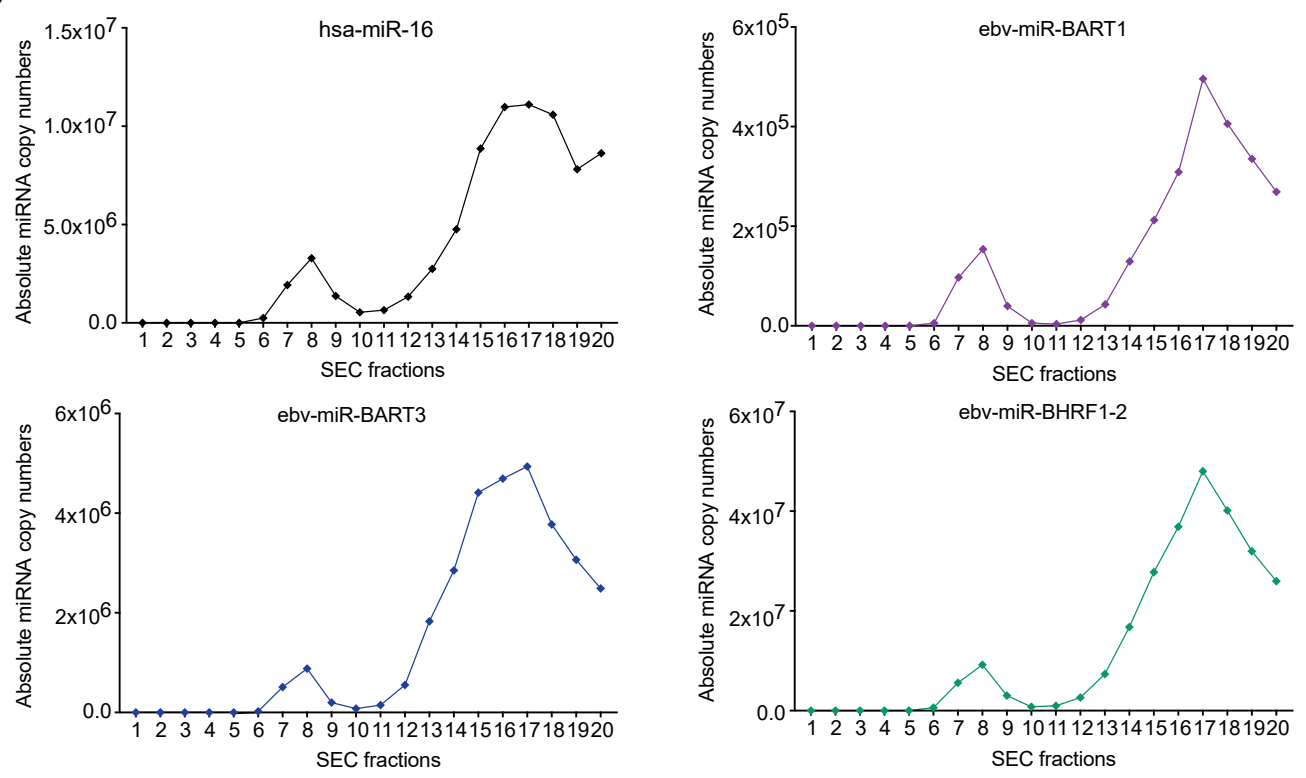

Figure 3 
A

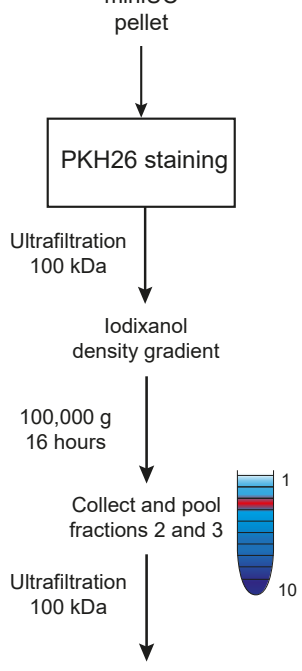

Stained EVs
B

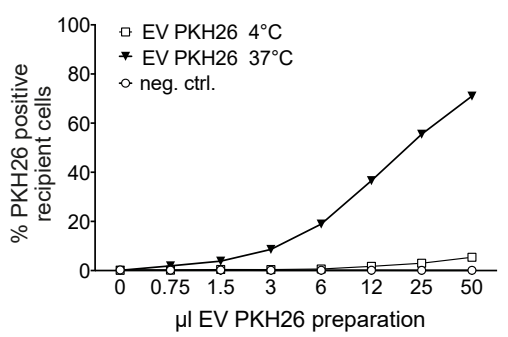

D

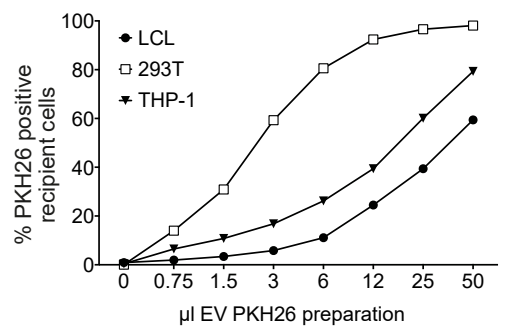

\section{C}

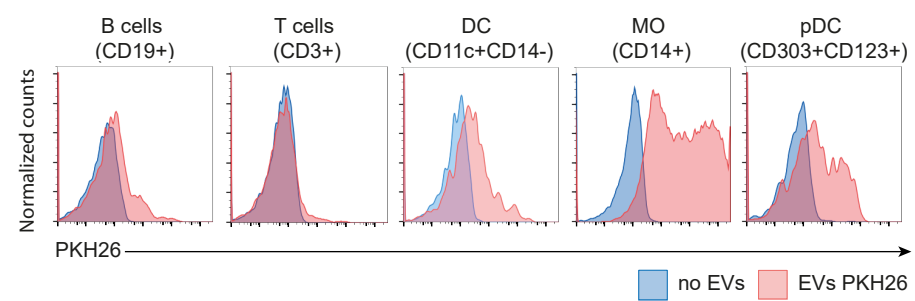

Figure 4 
A Donor cells CD63-BlaM positive with CD63-BlaM
Incubation of EVs with target cells
Staining with CCF4-AM

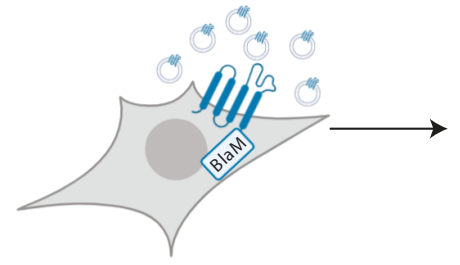

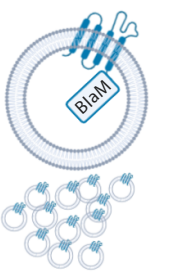

4 hours $37^{\circ} \mathrm{C}$

B

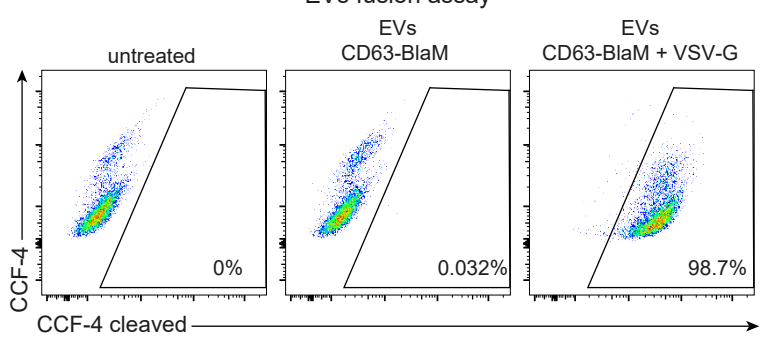

D

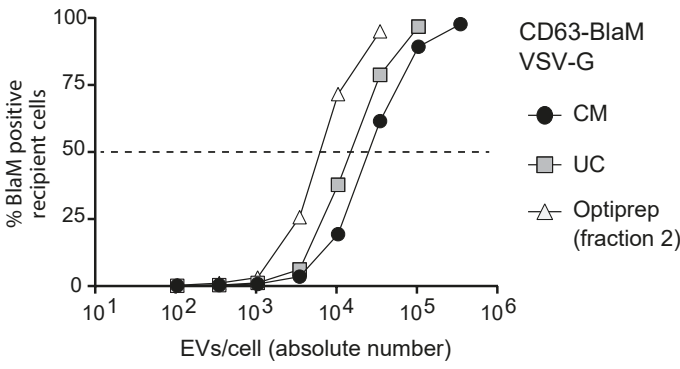

$\mathbf{F}$
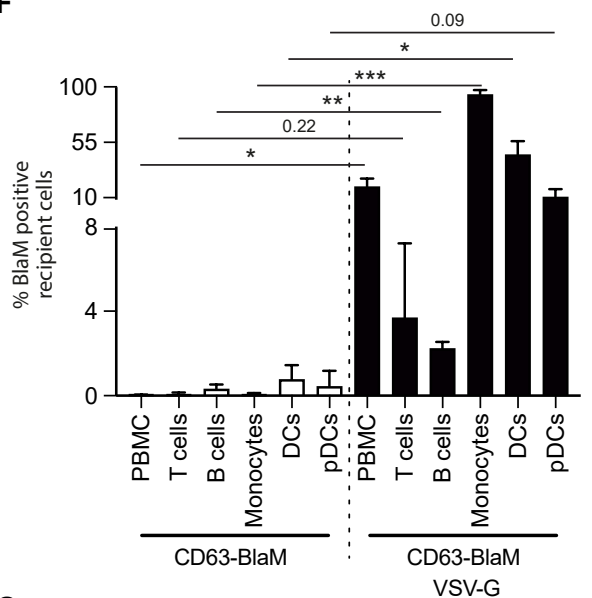

G

EVs VSV-G fusion in MDA-MB-231 cell lines

CCF-4

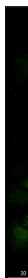

cleaved CCF-4

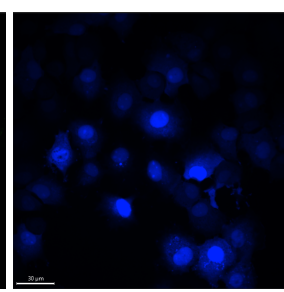

merged

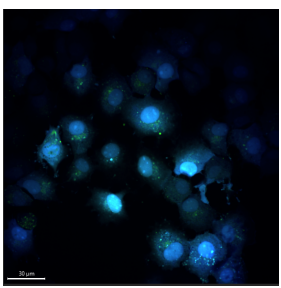

E
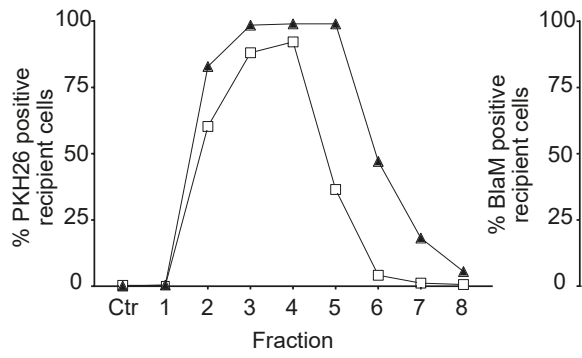

EVs CD63-BlaM

\section{Donor cells}

EVs without VSV-G

EVs with VSV-G

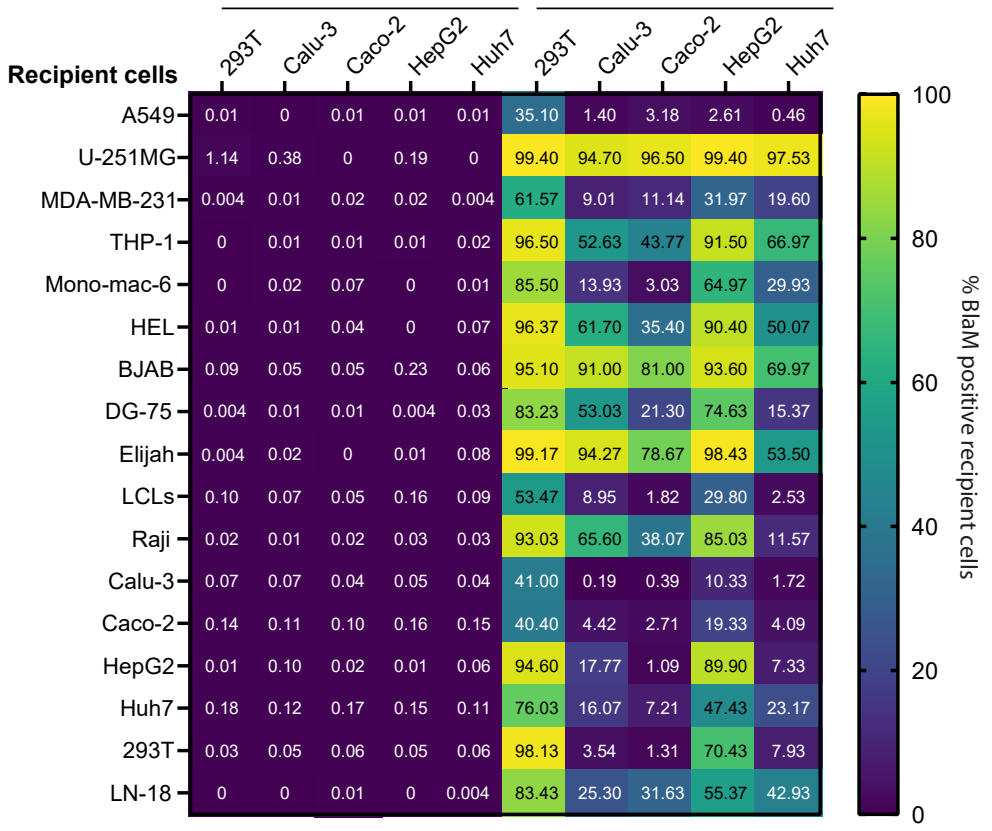

EVs VSV-G fusion in U-251MG cell lines

CCF-4

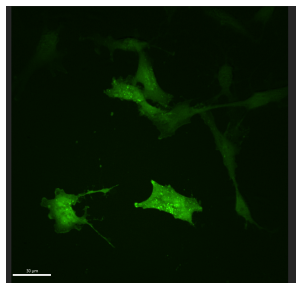

cleaved CCF-4

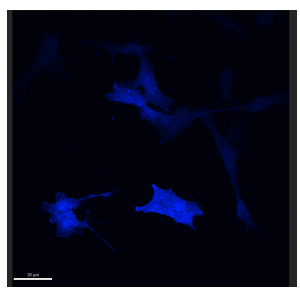

merged

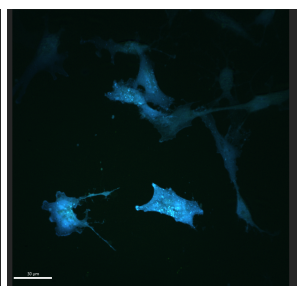

Figure 5 


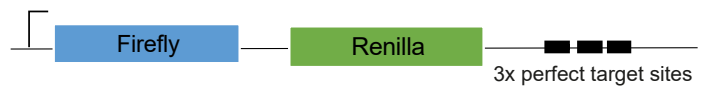

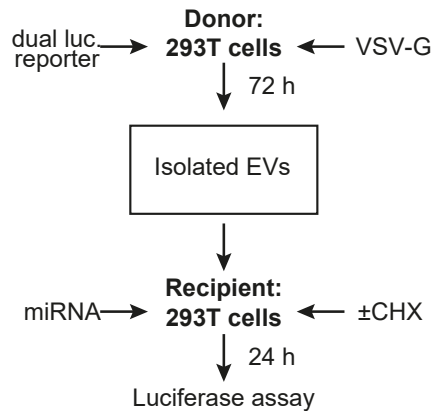

C

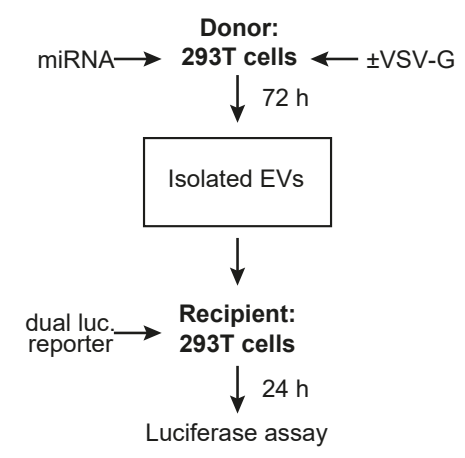
Luciferase assay
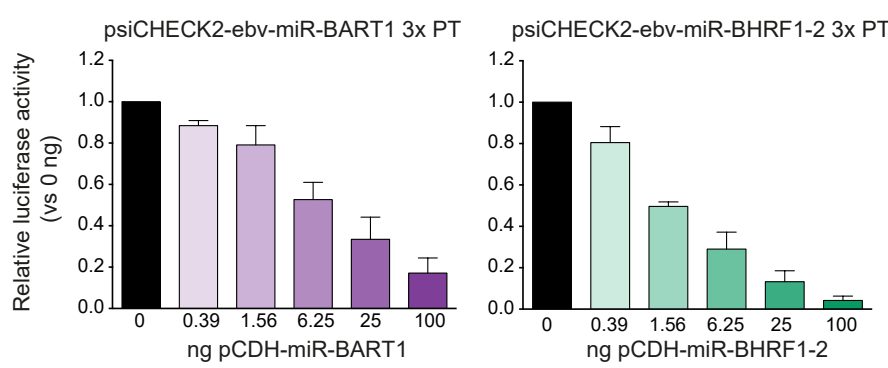

B
Recipient cells expressing miR-BART1

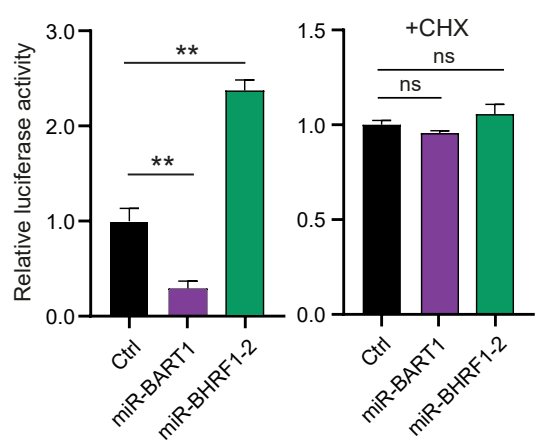

Recipient cells with miR-BART1 luciferase reporter

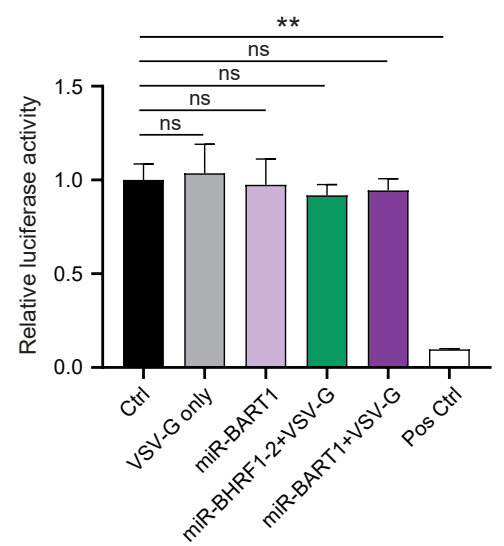

Recipient cells expressing miR-BHRF1-2
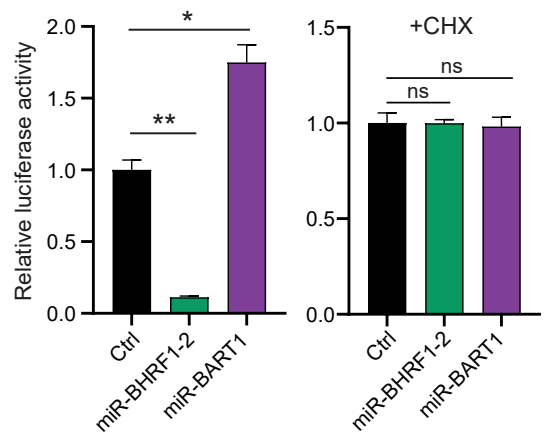

Recipient cells

with miR-BHRF1-2 luciferase reporter

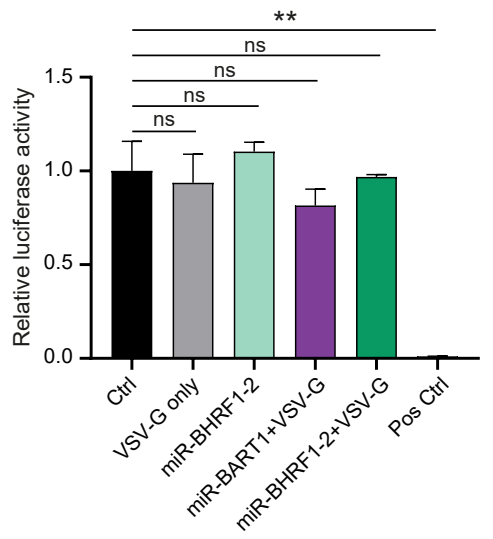

Figure 6 
A

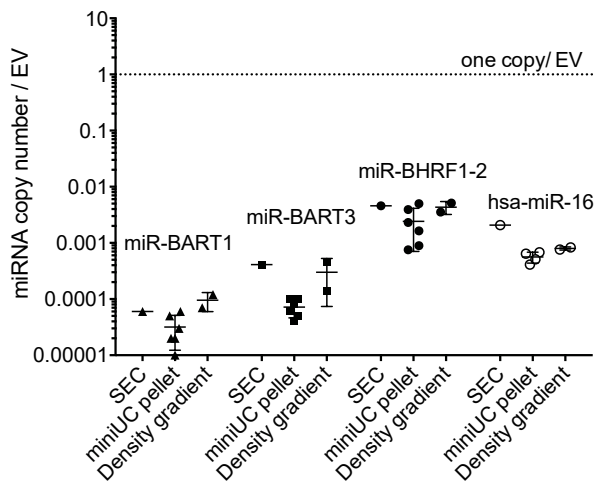

B

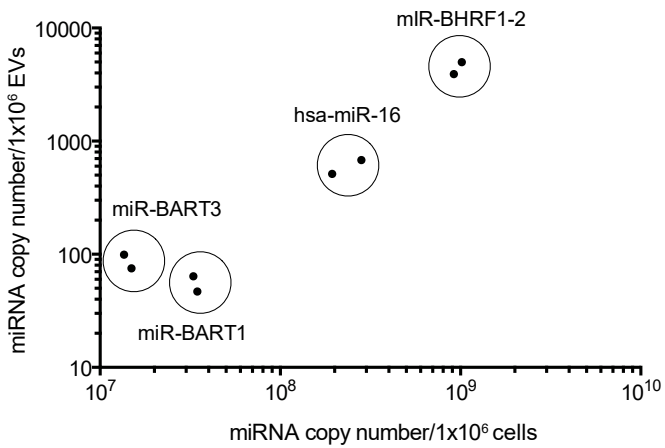

Figure 7 